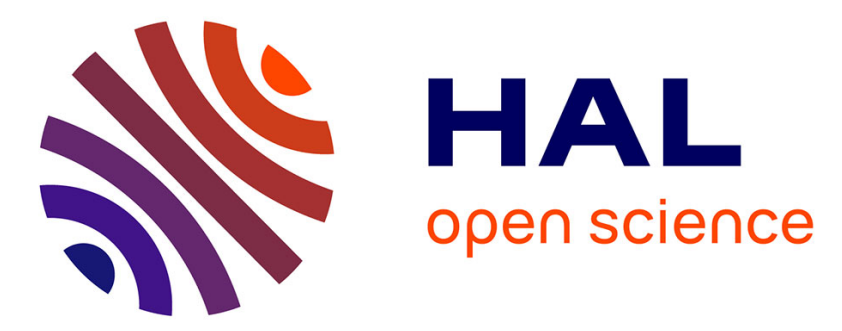

\title{
O VI in the Local Interstellar Medium
}

M. A. Barstow, D. D. Boyce, J. K. Barstow, A. E. Forbes, B. Y. Welsh, Rosine Lallement, S. Preval

\section{To cite this version:}

M. A. Barstow, D. D. Boyce, J. K. Barstow, A. E. Forbes, B. Y. Welsh, et al.. O VI in the Local Interstellar Medium. The Astrophysical Journal, 2010, 723 (2), pp.1762-1786. 10.1088/0004$637 \mathrm{X} / 723 / 2 / 1762$. hal-00531351

\section{HAL Id: hal-00531351 \\ https://hal.science/hal-00531351}

Submitted on 10 Jul 2020

HAL is a multi-disciplinary open access archive for the deposit and dissemination of scientific research documents, whether they are published or not. The documents may come from teaching and research institutions in France or abroad, or from public or private research centers.
L'archive ouverte pluridisciplinaire HAL, est destinée au dépôt et à la diffusion de documents scientifiques de niveau recherche, publiés ou non, émanant des établissements d'enseignement et de recherche français ou étrangers, des laboratoires publics ou privés. 


\title{
O VI IN THE LOCAL INTERSTELLAR MEDIUM
}

\author{
M. A. Barstow ${ }^{1}$, D. D. Boyce ${ }^{1}$, B. Y. Welsh ${ }^{2}$, R. Lallement ${ }^{3}$, J. K. Barstow ${ }^{1,4}$, A. E. Forbes ${ }^{1}$, And S. Preval ${ }^{1}$ \\ ${ }^{1}$ Department of Physics and Astronomy, University of Leicester, University Road, Leicester LE1 7RH, UK \\ ${ }^{2}$ Experimental Astrophysics Group, Space Sciences Laboratory, University of California Berkeley, Berkeley, CA 94720, USA \\ ${ }^{3}$ Service d'Aéronomie du CNRS, 91371 Verrières-le-Buisson, France \\ ${ }^{4}$ Atmospheric, Oceanic and Planetary Physics, Department of Physics, Clarendon Laboratory, Parks Road, Oxford OX1 3PU, UK \\ Received 2009 March 4; accepted 2010 September 13; published 2010 October 26
}

\begin{abstract}
We report the results of a search for O VI absorption in the spectra of 80 hot DA white dwarfs observed by the FUSE satellite. We have carried out a detailed analysis of the radial velocities of interstellar and (where present) stellar absorption lines for the entire sample of stars. In approximately $35 \%$ of cases (where photospheric material is detected), the velocity differences between the interstellar and photospheric components were beneath the resolution of the FUSE spectrographs. Therefore, in $65 \%$ of these stars the interstellar and photospheric contributions could be separated and the nature of the $\mathrm{O}$ VI component unambiguously determined. Furthermore, in other examples, where the spectra were of a high signal-to-noise, no photospheric material was found and any $\mathrm{O}$ VI detected was assumed to be interstellar. Building on the earlier work of Oegerle et al. and Savage \& Lehner, we have increased the number of detections of interstellar O VI and, for the first time, compared their locations with both the soft X-ray background emission and new detailed maps of the distribution of neutral gas within the local interstellar medium. We find no strong evidence to support a spatial correlation between $\mathrm{O}$ VI and SXRB emission. In all but a few cases, the interstellar OvI was located at or beyond the boundaries of the local cavity. Hence, any $T \sim 300,000 \mathrm{~K}$ gas responsible for the $\mathrm{O}$ vi absorption may reside at the interface between the cavity and surrounding medium or in that medium itself. Consequently, it appears that there is much less $\mathrm{O}$ VI-bearing gas than previously stated within the inner rarefied regions of the local interstellar cavity.
\end{abstract}

Key words: ISM: clouds - ISM: general - ISM: structure - ultraviolet: ISM - ultraviolet: stars - white dwarfs

Online-only material: color figures

\section{INTRODUCTION}

The existence of the diffuse soft X-ray background (SXRB; Bowyer et al. 1968) implies that a substantial fraction of the Galactic disk, at least near the Sun, is filled with low-density hot gas (McCammon et al. 1983). This discovery has important implications for our understanding of the structure and ionization of the interstellar medium (ISM) and in subsequent decades further studies have been made of the diffuse background, with a full sky survey being conducted by the ROSAT mission (Snowden et al. 1995, 1998). The total soft X-ray emission has been interpreted as the sum of three distinct components; extragalactic, galactic halo, and Local Bubble (LB) hot gas contributions. Details of these models and the constraints on the location of the soft X-ray emitting gas deduced from a number of soft X-ray shadows are discussed by Snowden et al. $(1998,2000)$ and Kuntz \& Snowden (2000), indicating that the temperature of the local hot gas is $T \approx 10^{6} \mathrm{~K}$. If this gas is homogeneously distributed in the LB, the measured emission in any direction must be proportional to the path length through the hot gas to the surrounding dense, cool material. Therefore, it is possible to map the shape of the emitting region (Snowden et al. 1998). Among the main features are two large elongations toward opposite directions at high galactic latitudes. Their axis is tilted by about $20^{\circ}-30^{\circ}$ with respect to the galactic polar axis, coinciding with neutral gas-free "chimneys" found nearly perpendicular to the Gould belt (Sfeir et al. 1999; Welsh et al. 1999).

Complementary to the study of the diffuse SXRB are observations of absorption from the lithium-like $\mathrm{O}$ VI ion transition doublet in the far-ultraviolet at 1031.91 and $1037.62 \AA$, arising in the ISM (Rogerson et al. 1973; York 1974; Jenkins \& Meloy 1974; Jenkins 1978). Ionization of O v to O VI requires an en- ergy input of $114 \mathrm{eV}$. Since the local interstellar radiation field declines steeply at energies above this value (Vallerga 1998), it is very difficult to account for the presence of $\mathrm{O}$ VI with a photoionization model. Therefore, the best explanation is that the $\mathrm{O}$ VI arises from collisional ionization in gas at a temperature in excess of $2 \times 10^{5} \mathrm{~K}$. This proposition is supported by the relative breadth of the $\mathrm{O}$ VI absorption line profiles, compared to those of lower ionization potential, which are consistent with thermal broadening at this temperature. In this paper, we refer to the $T \sim 300,000 \mathrm{~K}$ material which may be responsible for O vi absorption as "transition temperature" gas to differentiate it from the "hot" 1,000,000 K X-ray emitting gas.

The evidence for the presence of hot gas in the LB seems to be strong. However, there are a number of other observational results that contradict this picture. Any hot gas in the local ISM should give rise to a diffuse emission which should be detected not just in the soft X-ray waveband, but also in the extreme ultraviolet. Analysis of the ROSAT Wide Field Camera (WFC) EUV sky survey data reveals that the diffuse EUV background is negligible compared to other sources (West et al. 1994). Similarly, attempts to detect EUV line emission from the hot gas in deep exposures with the Extreme-Ultraviolet Explorer (EUVE) spectrographs yielded only upper limits to the flux (Vallerga \& Slavin 1998). Both the ROSAT WFC and EUVE telescopes were optimized for the detection and observation of point sources rather than diffuse emission. In contrast, the Cosmic Hot Interstellar Plasma Spectrometer was specifically designed to detect the line emission from the putative hot interstellar gas component. Again, no background emission was detected, although very tight constraints were put on the possible plasma emission measure (Hurwitz et al. 2005) placing it an order of magnitude lower than implied by the soft X-ray 
fluxes. Either there is less hot gas than expected, and the SXRB has another source, or the hot gas is significantly depleted in iron relative to other heavy elements, much more than reported elsewhere for hot gas.

One of the surprise results from the ROSAT mission was the detection of X-ray emission from the comet Hayakutake (Lisse et al. 1996), subsequently interpreted as arising from charge transfer of heavy solar wind ions with cometary neutrals (Cravens 1997). This solar wind charge exchange (SWCX) mechanism should also be experienced by neutral atoms of interstellar origin within the solar system. De-excitation of charge-exchanged atoms should then generate diffuse soft Xray emission throughout the heliosphere (Cox 1998; Cravens 2000). Long-term enhancements in the background observed by ROSAT appear to be correlated with solar activity (Freyberg 1994, Cravens 2000), indicating that a fraction of the SXRB arises from the heliosphere rather than the LB. Lallement (2004) finds that the fraction of SXRB emission attributable to our local cavity is substantially lower than previously thought.

Nevertheless, recent measurements of $\mathrm{O}$ VI absorption in the spectra of hot white dwarfs observed by the Far-Ultraviolet Spectroscopic Explorer (FUSE) mission provide renewed evidence for the presence of significant quantities of hot gas in the LB (Oegerle et al. 2005; Savage \& Lehner 2006). These authors report positive detections of $\mathrm{O}$ VI absorption along several local lines of sight but, as pointed out in these papers, there is the problem of possible contamination from photospheric material in the hottest white dwarfs making interpretation of the spectral measurements difficult. Indeed, International Ultraviolet Explorer (IUE) and Hubble Space Telescope (HST) highresolution UV observations of DA white dwarfs show that high ionization species such as $\mathrm{N} \mathrm{v}$ are found in the photospheres of white dwarfs down to surprisingly low effective temperatures. Originally, resonance lines of $\mathrm{N} \mathrm{v}, \mathrm{C}$ IV, and Si IV detected in the IUE echelle spectrum of GD659 $\left(T_{\text {eff }}=35,500 \mathrm{~K}\right)$, reported by Holberg et al. (1995) were thought to be circumstellar in origin. However, a new measurement of the stellar radial velocity shows that these features actually arise in the photosphere (Holberg et al. 2000). The same resonance lines are also detected at the photospheric velocity of REJ1032+532 (Holberg et al. $1999 ; T_{\text {eff }}=46,330 \mathrm{~K}$ ) and photospheric $\mathrm{N} \mathrm{v}$ is seen in the HST/GHRS spectrum of REJ1614-085 (Holberg et al. 1997; $T_{\text {eff }}=38,500 \mathrm{~K}$ ), indicating that it is not possible to assume that high ionization species are not photospheric in the cooler (i.e., $\left.T_{\text {eff }}<50,000 \mathrm{~K}\right)$ hot DA stars.

An additional concern is the apparent absence of detections of other high-ionization interstellar species in the UV spectra of white dwarfs. At gas temperatures sufficiently high to populate $\mathrm{O} v \mathrm{v}$, we might also expect to see $\mathrm{NV}$ and $\mathrm{C}$ IV. The ratio of the populations of $\mathrm{NV}$ and $\mathrm{CIV}$ to $\mathrm{OVI}$ will depend on the gas temperature, but for typical interstellar structures this lies somewhere in the range 0.1-0.4 (Indebetouw \& Shull 2004), indicating that the $\mathrm{NV}$ and $\mathrm{CIV}$ resonance doublets should be present if $\mathrm{OVVI}_{\mathrm{VI}}$ is detected. However, if the $\mathrm{O}$ VI lines are weak, the $\mathrm{N} \mathrm{v}$ and $\mathrm{C}$ IV may fall below their detection threshold. From an observational point of view, there are no detections of interstellar N v or C IV along the lines of sight to any local white dwarf observed with IUE (Holberg et al. 1998). Furthermore, careful radial velocity analyses of a smaller but higher signalto-noise sample of DA white dwarfs studied with HST/STIS showed detections of both photospheric and circumstellar $\mathrm{N} \mathrm{v}$ and C IV (Barstow et al. 2003b; Bannister et al. 2003), but no interstellar features.
Savage \& Lehner (2006) take the reasonable position that if no stellar or circumstellar heavy element lines are detected in a spectrum then any detected $\mathrm{O}_{\mathrm{VI}}$ is interstellar in origin. Ruling out a photospheric component is straightforward, since there are lines of $\mathrm{C}$ III/IV, O IV, Si IV, P IV/V, and S IV/VI that are readily detectable in the FUSE band. It is harder to be sure that there is no circumstellar component because there are no important resonance lines in the FUSE band other than the O VI. Fortunately, there are existing data from IUE and HST for a number of stars that can be used to confirm the absence of circumstellar material (e.g., WD1314+293, WD1254+223) and the work of Bannister et al. (2003) indicates that circumstellar components are only present when there is also photospheric material in a given star.

When stellar metals are present, following Oegerle et al. (2005) and Savage \& Lehner (2006), the only way to unambiguously determine the nature of a detection of O VI in FUSE white dwarf spectra is to carry out a thorough analysis of the radial velocity of known interstellar and photospheric features for each individual star for comparison with the O VI velocity. Even then the difference between the interstellar and photospheric velocities must be greater or equal to the velocity resolution of the instrument. Few stars which have photospheric features have sufficient velocity separation between these and the ISM lines. Hence, even samples of several tens of objects, as studied by Oegerle et al. (2005) and Savage \& Lehner (2006) are whittled down to just a few good targets. Since the work reported in these papers was carried out, more observations have been added to the FUSE archive, increasing the number of stars available for study. Often multiple observations of a single target are available and can be co-added to improve the resultant signal-to-noise ratio $(\mathrm{S} / \mathrm{N})$. Furthermore, the archival data are now available with an improved version of the FUSE pipeline (version 3.2, compared to version 2.05 and version 1.8.7 used by Savage \& Lehner and Oegerle et al., respectively), which (hopefully) yields better quality data in terms of $\mathrm{S} / \mathrm{N}$ and calibration.

It is therefore timely to re-examine the incidence of $\mathrm{O} V \mathrm{VI}$ in hot white dwarfs observed by FUSE and where present investigate its nature. We report here on such a new study including a sample of 80 stars drawn from the FUSE archive. Of this group, we have detected $\mathrm{OVI}$ in 26 cases where there is either no confusing photospheric component or when the interstellar and photospheric velocities are well separated. When we compare the distribution of stars in this study with neutral gas density maps of the local ISM it appears that the majority of interstellar $\mathrm{O}$ vi detections appear to be located at the periphery of or outside the LB. Hence, there is little evidence for the presence of million ${ }^{\circ} \mathrm{K}$ gas within the LB. In this paper, we describe our analysis of the white dwarf sample in detail and consider the implications our results have for our understanding of the local ISM and the origin of the SXRB.

\section{THE WHITE DWARF SAMPLE}

We have surveyed all the hot DA white dwarfs $\left(T_{\text {eff }}>\right.$ 20,000 K) observed by FUSE to look for evidence of the presence of interstellar O VI absorption. The total sample comprises 95 stars and includes all public observations accessible in the FUSE data archive up to 2007 August 1 . However, while there are many observations of cooler DAs and also a substantial number of observations of He-rich DO white dwarfs, we have excluded these targets from our study for various reasons. In the DA stars, at temperatures below $\sim 20,000 \mathrm{~K}$, the far-UV continuum flux is severely attenuated by the Lyman line series 
absorption in the spectral range occupied by the $\mathrm{O}$ vi doublet which yields an $\mathrm{S} / \mathrm{N}$ below acceptable limits for all reasonable exposures. In some stars, the continuum flux is contaminated by the presence of absorption by interstellar $\mathrm{H}_{2}$, leading to confusion in identifying the lines we need to measure for this work or reducing the $\mathrm{S} / \mathrm{N}$ of the data below useful levels. In principle, the DO white dwarfs, which all have temperatures above $\sim 45,000 \mathrm{~K}$, constitute excellent targets for this work. However, there are often many high-ionization features present in these for which the atomic data are not very reliable making it hard to determine reliable radial velocities (see Barstow et al. 2007), in contrast to the DAs. In addition, these objects potentially contain significant abundances of Fe group elements with features distributed throughout the FUSE wavelength range which might significantly contaminate or mimic a weak detection of $\mathrm{O}$ VI. Our resulting target sample of 80 stars has considerable overlap with both those of Oegerle et al. (2005) and Savage \& Lehner (2006), but is factors 3.6 and 1.7 larger, respectively. It is important to note that, because of the selection criteria we have outlined above, we have not included all the stars examined by these authors in this paper. On the other hand, they excluded from their analyses previously known stars where the $\mathrm{O}$ VI is thought to be contaminated or where the $\mathrm{S} / \mathrm{N}$ is not adequate, which we do consider here.

All the white dwarfs we have considered in our detailed analysis are listed in Table 1, along with their physical parameters ( $T_{\text {eff }}, \log g$, mass, and distance), where known, and their galactic coordinates. The targets include both isolated white dwarfs and also binary systems where the white dwarf has a mainsequence companion (mostly those objects with an HD number). For several binaries, no published white dwarf temperatures and gravities exist, so we have estimated these values from the Lyman lines present in the FUSE spectra, following the methods outlined in Barstow et al. (2003a). We have also updated some values where original estimates were made using just the Ly $\alpha$ line in IUE SWP spectra. The list is ordered by the white dwarf R.A/decl. number, where one has been assigned in the McCook \& Sion (1999) catalog, and any principal alternative name in general use is also given. Where there is no such name, the designation of the primary discovery survey is noted instead.

\section{OBSERVATIONS AND DATA REDUCTION}

\subsection{The FUSE Spectroscopic Data}

The FUSE mission was launched in 1999 and was successfully operated on-orbit until 2007. The instrument design and in-flight performance have been described in several scientific papers, including Moos et al. (2000) and Sahnow et al. (2000). The most recent version of the data processing pipeline, CALFUSE version 3, is described by Dixon et al (2007). Briefly, the spectrographs are based on a Rowland circle design, comprising four separate optical paths (or channels). The channels must be co-aligned so that light from a single target properly illuminates all four channels to maximize the throughput of the instrument. Spectra from the four channels are recorded on two microchannel plate detectors, with an $\mathrm{SiC}$ and $\mathrm{LiF}$ spectrum on each. Each detector is divided into two functionally independent segments (A and B), separated by a small gap. Consequently, there are eight detector segment/spectrometer channel combinations to be dealt with in reducing the data. Maintaining the co-alignment of individual channels has been difficult in-orbit, mainly due to thermal effects. A target may completely miss an aperture for the whole or part of an observation, while being well centered in the others. Hence, in any given observation, not all of the channels may be available in the data. To minimize this problem, most observations have been conducted using the largest aperture available (LWRS, $30 \times 30 \mathrm{arcsec}$ ). This limits the spectral resolution to between 15,000 and 20,000 for early observations or 23,000 later in the program when the mirror focusing had been adjusted (Sahnow et al. 2000), compared to the 24,000-30,000 expected for the $1.25 \times 20$ arcsec HIRS aperture. Various spectra analyzed here were obtained through HIRS, MDRS, or LWRS apertures and in TIMETAG or HISTOGRAM mode. Table 2 summarizes the data sets used for each of the white dwarf spectra studied in this paper.

\subsection{Data Reduction}

All the data used in this work were obtained from the archive (see Table 2) after reprocessing using version 3.2 of the CALFUSE pipeline. The data supplied by the archive consist of a set of files corresponding to the separate exposures for each of the eight channel/detector segment combinations. Since the $\mathrm{S} / \mathrm{N}$ of these can be relatively poor and the wavelength binning $(\sim 0.006 \AA)$ over-samples the true resolution by a factor $\sim 10$, we have established a tried and tested procedure to combine all the spectra (see Barstow et al. 2003a). Initially all the spectra were re-binned to a $0.02 \AA$ pixel size for examination. Any strong interstellar absorption features present are used to verify that the wavelength scales for each exposure are well aligned. Individual exposures are then co-added to produce a single spectrum, weighting the individual spectra by their exposure time. This whole procedure was repeated for all eight detector/ channel side combinations.

In principle, since we are mainly concerned with the analysis of the $\mathrm{O}$ VI lines in this paper, it is not necessary to study any segments other than the $\mathrm{LiF} 1 \mathrm{~A}, \mathrm{SiC} 1 \mathrm{~A}, \mathrm{LiF} 2 \mathrm{~B}$, and $\mathrm{SiC} 2 \mathrm{~B}$, which encompass the O vi doublet. However, for this work we also need to make accurate measurements of the interstellar and photospheric velocities. Ideally, these would simply be obtained from photospheric and interstellar features immediately adjacent the O VI lines. Unfortunately, in many of the stars in our sample this is not practical, since there are no suitable lines. There may be no photospheric lines at all in the segment, or the interstellar lines are saturated and/ or contaminated with geocoronal emission preventing accurate determination of the line velocity.

The perceived radial velocity of the photospheric lines will be the combination of both the intrinsic radial velocity of the star and its gravitational redshift, the latter depending on the stellar mass and radius. This could be measured at other wavelengths for our program stars, but few reliable measurements are available for them in the literature. Both the $I U E$ echelle and HST/STIS spectrographs had extremely good wavelength calibration and provide the best data but only $\sim 25 \%$ of our sample have been observed by these instruments (see Barstow et al. 2003b; Bannister et al. 2003). Even in these stars, assigning a photospheric velocity to the FUSE data is a tricky issue because the FUSE wavelength scale zero point is not necessarily the same for each exposure and spectral segment due to flexure in the instrument and the general use of the largest LWRS aperture to compensate, which leads to a significant uncertainty in the positioning of a source within it. As a result, the accuracy of the FUSE wavelength scale is typically limited to $\sim 15 \mathrm{~km} \mathrm{~s}^{-1}$. However, comparisons between the IUE echelle and FUSE for GD71 indicate that this uncertainty could be as large as $\sim 20 \mathrm{~km} \mathrm{~s}^{-1}$ (Dobbie et al. 2005). Therefore, we must be careful 
Table 1

Summary of White Dwarfs Targets and their Physical Parameters

\begin{tabular}{|c|c|c|c|c|c|c|c|c|}
\hline WD No. & Alt. Name/Cat ${ }^{\mathrm{a}}$ & $L\left(^{\circ}\right)$ & $b\left(^{\circ}\right)$ & $d(\mathrm{pc})$ & Reference & $T_{\text {eff }}$ & $\log g$ & Reference \\
\hline WD0001+433 & REJ, EUVEJ & 113.90 & -18.44 & 99 & This work & 46205 & 8.85 & Marsh et al. 1997 \\
\hline WD0004+330 & GD2 & 112.48 & -28.69 & 99 & Vennes et al. 1997 & 47936 & 7.77 & Marsh et al. 1997 \\
\hline WD0027-636 & REJ, EUVEJ & 306.98 & -53.55 & 236 & Vennes et al. 1997 & 60595 & 7.97 & Marsh et al. 1997 \\
\hline WD0050-332 & GD659 & 299.14 & -84.12 & 58 & Holberg et al. 1998 & 34684 & 7.89 & Marsh et al. 1997 \\
\hline WD0106-358 & GD683 & 280.88 & -80.81 & 86 & This work & 28580 & 7.90 & Marsh et al. 1997 \\
\hline WD0131-164 & GD984 & 167.26 & -75.15 & 96 & Vennes et al. 1997 & 44850 & 7.96 & Marsh et al. 1997 \\
\hline WD0147+674 & REJ, EUVEJ & 128.58 & 5.44 & 99 & Dupuis et al. 2005 & 30120 & 7.70 & Marsh et al. 1997 \\
\hline WD0226-615 & HD15638 & 284.20 & -52.16 & 199 & ESA 1997 & 50000 & 8.15 & Landsman et al. 1993 \\
\hline WD0229-481 & REJ, EUVEJ & 266.62 & -61.59 & 240 & This work & 63400 & 7.43 & Marsh et al. 1997 \\
\hline WD0232+035 & Feige 24 & 165.97 & -50.27 & 74 & ESA 1997 & 62947 & 7.53 & Marsh et al. 1997 \\
\hline WD0236+498 & REJ, EUVEJ & 140.15 & -9.15 & 96 & Vennes et al. 1997 & 33822 & 8.47 & Finley et al. 1997 \\
\hline WD0235-125 & PHL1400 & 165.97 & -50.27 & 86 & This work & 32306 & 8.44 & Finley et al. 1997 \\
\hline WD0252-055 & HD18131 & 181.86 & -53.47 & 104 & ESA 1997 & 29120 & 7.50 & Vennes et al. 1997 \\
\hline WD0320-539 & LB1663 & 267.30 & -51.64 & 124 & This work & 32860 & 7.66 & Marsh et al. 1997 \\
\hline WD0325-857 & REJ, EUVEJ & 299.86 & -30.68 & 35 & Barstow et al. 1995 & & & \\
\hline WD0346-011 & GD50 & 188.95 & -40.10 & 29 & Vennes et al. 1997 & 42373 & 9.00 & Marsh et al. 1997 \\
\hline WD0353+284 & REJ, EUVEJ & 165.08 & -18.67 & 107 & Burleigh et al. 1997 & 32984 & 7.87 & This work \\
\hline WD0354-368 & & 238.64 & -49.98 & 400 & Christian et al. 1996 & 53000 & 8.00 & Christian et al. 1996 \\
\hline WD0455-282 & REJ, EUVEJ & 229.29 & -36.17 & 102 & Oegerle et al. 2005 & 58080 & 7.90 & Marsh et al. 1997 \\
\hline WD0501+524 & G191-B2B & 155.95 & 7.10 & 59 & Vennes et al. 1997 & 57340 & 7.48 & Marsh et al. 1997 \\
\hline WD0512+326 & HD33959C & 173.30 & -3.36 & 25 & ESA 1997 & 22750 & 8.01 & Holberg et al. 1999 \\
\hline WD0549+158 & GD71 & 192.03 & -5.34 & 49 & Vennes et al. 1997 & 32780 & 7.83 & Barstow et al. 2003a \\
\hline WD0603-483 & REJ, EUVEJ & 255.78 & -27.36 & 178 & Vennes et al. 1997 & 33040 & 7.80 & Marsh et al. 1997 \\
\hline WD0621-376 & REJ, EUVEJ & 245.41 & -21.43 & 78 & Holberg et al. 1998 & 62280 & 7.22 & Marsh et al. 1997 \\
\hline WD0659+130 & REJ0702+129 & 202.51 & 8.19 & 115 & Vennes et al. 1998 & 39960 & 8.31 & This work \\
\hline WD0715-704 & REJ, EUVEJ & 281.40 & -23.50 & 94 & Vennes et al. 1997 & 44300 & 7.69 & Marsh et al. 1997 \\
\hline WD0802+413 & KUV & 179.22 & 30.94 & 230 & Dupuis et al. 2005 & 45394 & 7.39 & Finley et al. 1997 \\
\hline WD0830-535 & REJ, EUVEJ & 270.11 & -8.27 & 82 & Vennes et al. 1997 & 29330 & 7.79 & Marsh et al. 1997 \\
\hline WD0937+505 & PG & 166.90 & 47.12 & 218 & Dupuis et al. 2005 & 35552 & 7.76 & Finley et al. 1997 \\
\hline WD1019-141 & REJ, EUVEJ & 256.48 & 34.74 & 112 & This work & 29330 & 7.79 & Marsh et al. 1997 \\
\hline WD1021+266 & HD90052 & 205.72 & 57.21 & 250 & Burleigh et al. 1997 & 35432 & 7.48 & This work \\
\hline WD1024+326 & REJ, EUVEJ & 194.52 & 58.41 & 400 & This work & 41354 & 7.59 & This work \\
\hline WD1029+537 & REJ, EUVEJ & 157.51 & 53.24 & 116 & Vennes et al. 1997 & 44980 & 7.68 & Marsh et al. 1997 \\
\hline WD1040+492 & REJ, EUVEJ & 162.67 & 57.01 & 230 & Vennes et al. 1997 & 47560 & 7.62 & Marsh et al. 1997 \\
\hline WD1041+580 & REJ, EUVEJ & 150.12 & 52.17 & 93 & Vennes et al. 1997 & 29016 & 7.79 & Marsh et al. 1997 \\
\hline WD1057+719 & PG & 134.48 & 42.92 & 141 & Savage \& Lehner 2006 & 39555 & 7.66 & Marsh et al. 1997 \\
\hline WD1109-225 & HD97277 & 274.78 & 34.54 & 82 & ESA 1997 & 36750 & 7.50 & Barstow et al. 1994 \\
\hline WD1234+481 & PG & 129.81 & 69.01 & 129 & Vennes et al. 1997 & 55570 & 7.57 & Marsh et al. 1997 \\
\hline WD1254+223 & GD153 & 317.25 & 84.75 & 67 & Vennes et al. 1997 & 38205 & 7.90 & Barstow et al. 2003a \\
\hline WD1302+597 & GD323 & 119.82 & 57.59 & 79 & This work & 39960 & 8.31 & This work \\
\hline WD1314+293 & $\mathrm{HZ} 43$ & 54.11 & 84.16 & 68 & Finley et al. 1997 & 49435 & 7.95 & Barstow et al. 2003a \\
\hline WD1337+701 & EG102 & 117.18 & 46.31 & 104 & Dupuis et al. 2005 & 20435 & 7.87 & Finley et al. 1997 \\
\hline WD1342+442 & PG & 94.30 & 69.92 & 387 & This work & 66750 & 7.93 & Barstow et al. 2003a \\
\hline WD1440+753 & REJ, EUVEJ & 114.10 & 40.12 & 98 & Vennes et al. 1997 & 42400 & 8.54 & Vennes et al. 1997 \\
\hline WD1528+487 & REJ, EUVEJ & 78.87 & 52.72 & 140 & Vennes et al. 1997 & 46230 & 7.70 & Marsh et al. 1997 \\
\hline WD1550+130 & NN Ser & 23.65 & 45.34 & 756 & This work & 39910 & 6.82 & This work \\
\hline WD1603+432 & PG & 68.22 & 47.93 & 114 & Dupuis et al. 2005 & 36257 & 7.85 & Finley et al. 1997 \\
\hline WD1611-084 & REJ, EUVEJ & 4.30 & 29.30 & 93 & Vennes 1999 & 38500 & 7.85 & Marsh et al. 1997 \\
\hline WD1615-154 & G153-41 & 358.79 & 24.18 & 55 & Oegerle et al. 2005 & 38205 & 7.90 & Barstow et al. 2003a \\
\hline WD1620+647 & PG & 96.61 & 40.16 & 174 & Dupuis et al. 2005 & 30184 & 7.72 & Finley et al. 1997 \\
\hline WD1631+781 & REJ, EUVEJ & 111.30 & 33.58 & 67 & Vennes et al. 1997 & 44559 & 7.79 & Finley et al. 1997 \\
\hline WD1635+529 & HD150100 & 80.94 & 41.49 & 123 & ESA 1997 & 20027 & 8.14 & This work \\
\hline WD1636+351 & WD1638+349 & 56.98 & 41.40 & 109 & Vennes et al. 1997 & 36056 & 7.71 & Marsh et al. 1997 \\
\hline WD1648+407 & REJ, EUVEJ & 64.64 & 39.60 & 200 & Vennes et al. 1997 & 37850 & 7.95 & Marsh et al. 1997 \\
\hline WD $1725+586$ & LB335 & 87.17 & 33.83 & 123 & This work & 54550 & 8.49 & Marsh et al. 1997 \\
\hline WD1800+685 & KUV & 98.73 & 29.78 & 159 & Vennes et al. 1997 & 43701 & 7.80 & Marsh et al. 1997 \\
\hline WD1819+580 & REJ, EUVEJ & 87.00 & 26.76 & 103 & This work & 45330 & 7.73 & Marsh et al. 1997 \\
\hline WD1844-223 & REJ, EUVEJ & 12.50 & -9.25 & 62 & Vennes et al. 1997 & 31470 & 8.17 & Finley et al. 1997 \\
\hline WD1845+683 & KUV & 98.81 & 25.65 & 125 & Vennes et al. 1997 & 36888 & 8.12 & Finley et al. 1997 \\
\hline WD1917+509 & REJ, EUVEJ & 91.02 & 20.04 & 105 & Vennes et al. 1997 & 33000 & 7.90 & Vennes et al. 1997 \\
\hline WD1921-566 & REJ, EUVEJ & 340.63 & -27.01 & 110 & Vennes et al. 1998 & 52946 & 8.16 & This work \\
\hline WD1942+499 & & 83.08 & 12.75 & 105 & Vennes et al. 1997 & 33500 & 7.86 & Marsh et al. 1997 \\
\hline WD1950-432 & HS & 356.51 & -29.09 & 140 & Dupuis et al. 2005 & 41339 & 7.85 & Finley et al. 1997 \\
\hline WD2000-561 & REJ, EUVEJ & 341.78 & -32.25 & 198 & Dupuis et al. 2005 & 44456 & 7.54 & Marsh et al. 1997 \\
\hline WD2004-605 & REJ, EUVEJ & 336.58 & -32.86 & 58 & Vennes et al. 1997 & 44200 & 8.14 & Marsh et al. 1997 \\
\hline WD2011+398 & REJ, EUVEJ & 77.00 & 3.18 & 141 & Vennes et al. 1997 & 47057 & 7.74 & Marsh et al. 1997 \\
\hline
\end{tabular}


Table 1

(Continued)

\begin{tabular}{|c|c|c|c|c|c|c|c|c|}
\hline WD No. & Alt. Name/Cat ${ }^{\mathrm{a}}$ & $L\left(^{\circ}\right)$ & $b\left(^{\circ}\right)$ & $d(\mathrm{pc})$ & Reference & $T_{\text {eff }}$ & $\log g$ & Reference \\
\hline WD2014-575 & LS210-114 & 340.20 & -34.25 & 51 & Vennes et al. 1997 & 26579 & 7.78 & Marsh et al. 1997 \\
\hline WD2020-425 & REJ, EUVEJ & 358.36 & -34.45 & 52 & This work & 28597 & 8.54 & Marsh et al. 1997 \\
\hline WD2111+498 & GD394 & 91.37 & 1.13 & 50 & Vennes et al. 1997 & 38866 & 7.84 & Marsh et al. 1997 \\
\hline WD2116+736 & KUV & 109.39 & 16.92 & 177 & Dupuis et al. 2005 & 54486 & 7.76 & Finley et al. 1997 \\
\hline WD2124+191 & HD204188 & 70.43 & -21.98 & 46 & Vennes et al. 1998 & 35000 & 9.00 & Landsman et al. 1993 \\
\hline WD2124-224 & REJ, EUVEJ & 27.36 & -43.76 & 224 & Oegerle et al. 2005 & 48297 & 7.69 & Marsh et al. 1997 \\
\hline WD2146-433 & REJ, EUVEJ & 357.18 & -50.13 & 362 & Dupuis et al. 2005 & 67912 & 7.58 & Finley et al. 1997 \\
\hline WD2152-548 & REJ, EUVEJ & 340.50 & -48.70 & 129 & $\ldots$ & 45800 & 7.78 & Vennes et al. 1997 \\
\hline WD2211-495 & REJ, EUVEJ & 345.79 & -52.62 & 53 & Vennes et al. 1997 & 65600 & 7.42 & Marsh et al. 1997 \\
\hline WD2257-073 & HD217411 & 65.17 & -56.93 & 89 & Vennes et al. 1998 & 38010 & 7.84 & This work \\
\hline WD2309+105 & GD246 & 87.26 & -45.12 & 79 & Vennes et al. 1997 & 51300 & 7.91 & Barstow et al. 2003a \\
\hline WD2321-549 & REJ, EUVEJ & 326.91 & -58.21 & 192 & Dupuis et al. 2005 & 45860 & 7.73 & Marsh et al. 1997 \\
\hline WD2331-475 & REJ, EUVEJ & 334.84 & -64.81 & 82 & Vennes et al. 1997 & 56682 & 7.64 & Marsh et al. 1997 \\
\hline WD2350-706 & HD223816 & 309.91 & -45.94 & 92 & Barstow et al. 2001 & 69300 & 8.00 & Barstow et al. 1994 \\
\hline
\end{tabular}

Note. ${ }^{\text {a }}$ PG, Palomar Green; HS: Hamburg Schmidt; REJ, ROSAT EUV; EUVEJ, EUVE; KUV, Kiso UV; KPD, Kitt Peak Downes.

when directly comparing a velocity reference from IUE or $H S T$ with a FUSE measurement of an O VI velocity.

For the purposes of this analysis we do not actually need a very accurate absolute measurement of the radial velocities. What is most important is that we are able to obtain an accurate measurement of the difference between the interstellar and stellar components and an accurate determination of the $\mathrm{O}$ VI velocity on the same scale. Any undetermined offset to the wavelength zero point would be unimportant provided it is the same for all data segments. Therefore, to be able to obtain good relative velocities it was necessary to combine the individual spectral segments to provide continuous coverage across the full FUSE spectrum. First, all the spectra were re-sampled onto a common wavelength scale of $0.02 \AA$ steps to reduce the level of oversampling. The re-sampled/re-binned spectra were then co-added, weighting individual data points by the statistical variance, averaged over a $20 \AA$ interval, to take into account any differences in the effective area of each segment and any differences in the exposure time that may have arisen from rejection of bad data segments (e.g., those with reduced exposure due to source drift). We have used the measured wavelengths of the strongest unsaturated interstellar lines to adjust the wavelength scale for each segment before co-addition, taking the $\mathrm{LiF} 1 \mathrm{~A}$ as our reference point against which every other segment is adjusted.

Through visual inspection, it is apparent that the statistical noise tends to increase toward the edges of a wavelength range. In cases where the $\mathrm{S} / \mathrm{N}$ is particularly poor $(<3: 1$ per resolution element) in these regions, we have trimmed the spectra (by $\sim 2-3 \AA$ ) to remove these data points prior to co-addition. An example of the resulting spectra, taking one of the higher $\mathrm{S} / \mathrm{N}$ data sets, is shown in Figure 1. The region of poor $\mathrm{S} / \mathrm{N}$ seen in the $1080-1087 \AA$ region is due to the absence of $\mathrm{LiF}$ data at those wavelengths. Where we had multiple exposures for any white dwarf, we carried out a further stage of evaluation of the spectra, discarding those where the $\mathrm{S} / \mathrm{N}$ was particularly poor or where the flux levels appeared to be anomalous, when compared to the remainder.

\subsection{Measuring Photospheric and Interstellar Radial Velocities}

The output of our data reduction procedure is a composite, wavelength calibrated spectrum covering the full range of the FUSE spectrographs for each white dwarf in the sample. The data for individual stars can then be visually searched for absorption features across the full wavelength range. In a separate exercise, we have cross-correlated the measured wavelengths of detected features with all the publicly available line lists (e.g., Kurucz \& Bell 1995; Morton 2003; Kentucky database—http://www.pa.uky.edu/ peter/atomic). A particular problem for line identification in the far-UV is the large number of possible transitions for feasible species, often leading to several alternative nearby identifications (i.e., within the measurement and wavelength scale uncertainties) for a single feature. By requiring that all reliable identifications correspond to one of two possible radial velocities, either interstellar or photospheric, in an individual star and searching for similar absorption patterns in a number of stars, we have produced a master list for line identification purposes (Boyce 2009). This forms the basis for the determination of interstellar and stellar radial velocities in this paper.

For each star in the sample, we have searched for significant features across the full FUSE range and measured their wavelengths using a simple determination of the centroid of the feature by fitting a Gaussian profile. In general, it is not necessary or appropriate to use more sophisticated line fitting procedures due to the undersampling of the lines by the FUSE spectral resolution and the relatively low $\mathrm{S} / \mathrm{N}$ of most features. The main purpose of this paper is to compare the observed velocity of any $\mathrm{O}$ VI features detected in these white dwarf spectra with the interstellar and stellar velocities to determine with which particular component, if any, it is associated. Ideally, to avoid any issues associated with differing wavelength calibrations for different spectroscopic segments, the comparison should be against lines that are close to $\mathrm{O}$ VI in wavelength and in the same spectral segment. The available interstellar reference lines are C II (1036.337 $)$, O I (1039.230 ̊), and Ar I $(1048.220 \AA)$, but there are few potential photospheric lines in the region apart from the O vi itself. Several P IV lines (1030.515, 1033.11, and 1035.516) fall in this wavelength range, but are present in only a few stars. Furthermore, any O I absorption may be contaminated by geocoronal emission and the $\mathrm{C}$ II can be saturated, compromising the wavelength measurement in either case. If we were to rely on velocity comparisons in the $\mathrm{O}$ vi region alone, we would have very few stars available for this study. Therefore, we have determined the mean interstellar and stellar velocities for each star from all the relevant lines available across the full FUSE spectral range. These results are summarized in Figures 2 and 3, and Table 3, together with the 
Table 2

Summary of FUSE Observations

\begin{tabular}{|c|c|c|c|}
\hline WD No. & Alt. Name/Cat & Observation & Start Time (yyyy-mm-dd) \\
\hline WD0001+433 & REJ, EUVEJ & E90305010 & 2004-11-24 19:34:00 \\
\hline WD0004+330 & GD2 & P20411020-40 & 2002-09-14 10:07:00 \\
\hline WD0027-636 & REJ, EUVEJ & Z90302010 & 2002-09-30 15:44:00 \\
\hline \multirow[t]{2}{*}{ WD0050-332 } & \multirow[t]{2}{*}{ GD659 } & M10101010 & 2000-07-04 23:52:00 \\
\hline & & P20420010 & 2000-12-11 07:00:00 \\
\hline WD0106-358 & \multirow[t]{2}{*}{ GD683 } & D02301010 & 2004-11-21 06:29:00 \\
\hline WD0111+002 & & A01303030 & 2001-08-07 12:43:00 \\
\hline WD0131-164 & GD984 & P20412010 & 2000-12-10 07:45:00 \\
\hline WD0147+674 & REJ, EUVEJ & Z90303020 & 2002-10-12 17:08:00 \\
\hline WD0226-615 & HD15638 & A05402010 & 2000-07-04 20:39:00 \\
\hline WD0229-481 & REJ, EUVEJ & M10504010 & 2002-09-21 11:51:00 \\
\hline WD0232+035 & Feige 24 & P10405040 & 2004-01-06 01:00:00 \\
\hline WD0236+498 & REJ, EUVEJ & Z90306010 & 2002-12-11 16:47:00 \\
\hline WD0235-125 & PHL1400 & E56809010 & 2004-08-30 17:27:00 \\
\hline WD0252-055 & HD18131 & A05404040 & 2001-11-28 06:39:00 \\
\hline WD0320-539 & LB1663 & D02302010 & 2003-09-09 08:50:00 \\
\hline WD0325-857 & REJ, EUVEJ & A01001010 & 2000-09-17 19:52:00 \\
\hline WD0346-011 & GD50 & B12201020-50 & 2003-12-20 04:43:00 \\
\hline WD0353+284 & REJ, EUVEJ & B05510010 & 2001-01-02 22:31:00 \\
\hline WD0354-368 & & B05511010 & 2001-08-12 11:18:00 \\
\hline WD0416+402 & KPD & Z90308010 & 2003-10-05 13:39:00 \\
\hline WD0455-282 & REJ, EUVEJ & P10411030 & 2000-02-07 09:36:00 \\
\hline WD0501+524 & G191-B2B & Coadded $*$ & \\
\hline WD0512+326 & HD33959C & A05407070 & 2000-03-01 04:08:00 \\
\hline WD0549+158 & GD71 & P20417010 & 2000-11-04 15:32:00 \\
\hline WD0603-483 & REJ, EUVEJ & Z90309010 & 2002-12-31 19:26:00 \\
\hline WD0621-376 & REJ, EUVEJ & P10415010 & 2000-12-06 05:28:00 \\
\hline WD0659+130 & REJ0702+129 & B05509010 & 2001-03-05 21:53:00 \\
\hline WD0715-704 & REJ, EUVEJ & M10507010 & 2003-08-15 00:08:00 \\
\hline WD0802+413 & KUV & Z90311010 & 2004-03-14 09:37:00 \\
\hline WD0830-535 & REJ, EUVEJ & Z90313010 & 2002-05-03 13:37:00 \\
\hline WD0937+505 & PG & Z90314010 & 2003-03-24 09:13:00 \\
\hline WD1019-141 & REJ, EUVEJ & P20415010 & 2001-05-15 21:35:00 \\
\hline WD1021+266 & HD90052 & B05508010 & 2001-05-01 19:34:00 \\
\hline WD1024+326 & REJ, EUVEJ & B05512010 & 2001-05-01 09:22:00 \\
\hline WD1029+537 & REJ, EUVEJ & B00301010 & 2001-03-25 04:40:00 \\
\hline WD1040+492 & REJ, EUVEJ & Z00401010 & 2002-04-04 12:42:00 \\
\hline WD1041+580 & REJ, EUVEJ & Z90317010 & 2002-04-07 10:04:00 \\
\hline WD1057+719 & PG & Z90318010 & 2002-04-08 16:12:00 \\
\hline WD1109-225 & HD97277 & A05401010 & 2000-05-29 16:00:00 \\
\hline WD1234+481 & PG & M10524020 & 2003-03-18 20:29:00 \\
\hline WD1254+223 & GD153 & M10104020 & 2000-04-29 21:32:00 \\
\hline WD1302+597 & GD323 & C02601010 & 2002-04-02 17:00:00 \\
\hline WD1314+293 & HZ43 & P10423010 & 2000-04-22 21:19:00 \\
\hline WD1337+701 & EG102 & B11903010 & 2001-05-05 06:43:00 \\
\hline WD1342+442 & PG & A03404020 & 2000-01-11 23:01:00 \\
\hline WD1440+753 & REJ, EUVEJ & Z90322010 & 2003-01-15 08:40:00 \\
\hline WD1528+487 & REJ, EUVEJ & P20401010 & 2001-03-27 23:14:00 \\
\hline WD1550+130 & NN Ser & E11201010 & 2004-04-15 05:38:00 \\
\hline WD1603+432 & PG & Z90324010 & 2003-07-02 11:51:00 \\
\hline WD1611-084 & REJ, EUVEJ & B11904010 & 2004-04-02 11:53:00 \\
\hline WD1615-154 & G153-41 & P20419010 & 2001-08-29 12:40:00 \\
\hline WD1620+647 & PG & Z90325010 & 2002-05-17 04:45:00 \\
\hline WD1631+781 & REJ, EUVEJ & M10528040 & 2004-02-13 20:02:00 \\
\hline WD1635+529 & HD150100 & C05002010 & 2002-07-13 14:00:00 \\
\hline WD1636+351 & WD1638+349 & P20402010 & 2001-03-28 14:22:00 \\
\hline WD1648+407 & REJ, EUVEJ & Z90326010 & 2002-07-12 11:22:00 \\
\hline WD1725+586 & LB335 & Z90327010 & 2002-05-13 23:16:00 \\
\hline \multirow[t]{2}{*}{ WD1800+685 } & \multirow[t]{2}{*}{ KUV } & M10530010-70 & 2002-09-09 15:14:53 \\
\hline & & P20410010-20 & 2001-10-01 04:55:36 \\
\hline WD1819+580 & REJ, EUVEJ & Z90328010 & 2002-05-11 01:25:00 \\
\hline WD1844-223 & REJ, EUVEJ & P20405010 & 2001-04-28 20:01:00 \\
\hline \multirow[t]{2}{*}{ WD1845+683 } & \multirow[t]{2}{*}{ KUV } & Z90329010 & 2002-05-08 20:40:00 \\
\hline & & Z99003010 & 2002-09-13 10:39:00 \\
\hline WD1917+509 & REJ, EUVEJ & Z90330010 & 2002-05-07 01:36:00 \\
\hline WD1921-566 & REJ, EUVEJ & A05411110 & 2000-05-24 22:59:00 \\
\hline WD1942+499 & & Z90331010 & 2002-05-08 00:12:00 \\
\hline
\end{tabular}


Table 2

(Continued)

\begin{tabular}{|c|c|c|c|}
\hline WD No. & Alt. Name/Cat & Observation & Start Time (yyyy-mm-dd) \\
\hline WD1950-432 & HS & Z90332010 & 2002-10-04 09:18:00 \\
\hline WD2000-561 & REJ, EUVEJ & Z90333010 & 2002-04-15 21:30:00 \\
\hline WD2004-605 & REJ, EUVEJ & P20422010 & 2001-05-21 21:09:00 \\
\hline WD2011+398 & REJ, EUVEJ & M10531020 & 2003-10-23 12:19:00 \\
\hline WD2014-575 & LS210-114 & Z90334010 & 2002-04-16 15:55:00 \\
\hline WD2020-425 & REJ, EUVEJ & Z90335010 & 2002-06-07 09:16:00 \\
\hline WD2043-635 & BPM13537 & Z90337010 & 2002-04-13 09:10:00 \\
\hline WD2111+498 & GD394 & M10532010 & 2002-10-27 16:44:00 \\
\hline WD2116+736 & KUV & Z90338020 & 2002-10-28 01:26:00 \\
\hline WD2124+191 & HD204188 & A05409090 & 2001-07-12 22:23:00 \\
\hline WD2124-224 & REJ, EUVEJ & P20406010 & 2001-10-08 20:26:00 \\
\hline WD2146-433 & REJ, EUVEJ & Z90339010 & 2002-10-06 15:14:00 \\
\hline WD2152-548 & REJ, EUVEJ & M10515010 & 2002-09-24 03:07:00 \\
\hline WD2211-495 & REJ, EUVEJ & M10303160 & 2003-05-24 23:28:00 \\
\hline WD2257-073 & HD217411 & A05410100 & 2000-06-28 11:05:00 \\
\hline WD2309+105 & GD246 & P20424010 & 2001-07-14 01:26:00 \\
\hline WD2321-549 & REJ, EUVEJ & Z90342010 & 2002-07-22 05:10:00 \\
\hline WD2331-475 & REJ, EUVEJ & M10517010 & 2002-09-23 08:46:00 \\
\hline \multirow[t]{50}{*}{ WD2350-706 } & HD223816 & A05408090 & 2001-05-23 16:21:00 \\
\hline & *G191-B2B obs. & M1010201000 & 1999-10-13 01:25:00 \\
\hline & & M1030501000 & 1999-11-12 07:35:00 \\
\hline & & M1030502000 & 1999-11-20 07:22:00 \\
\hline & & M1030401000 & 1999-11-20 09:02:00 \\
\hline & & M1030603000 & 1999-11-20 10:43:00 \\
\hline & & M1030503000 & 1999-11-21 06:43:00 \\
\hline & & M1030504000 & 1999-11-21 10:03:00 \\
\hline & & M1030602000 & 1999-11-21 11:39:00 \\
\hline & & S3070101000 & 2000-01-14 09:40:00 \\
\hline & & M1010202000 & 2000-02-17 06:10:00 \\
\hline & & M1030604000 & 2001-01-09 09:02:00 \\
\hline & & M1030506000 & 2001-01-09 09:26:00 \\
\hline & & M1030605000 & 2001-01-10 13:20:00 \\
\hline & & M1030507000 & 2001-01-10 13:45:00 \\
\hline & & M1030403000 & 2001-01-10 15:08:00 \\
\hline & & M1030606000 & 2001-01-23 06:08:00 \\
\hline & & M1030508000 & 2001-01-23 07:55:00 \\
\hline & & M1030404000 & 2001-01-23 11:18:00 \\
\hline & & M1030607000 & 2001-01-25 04:46:00 \\
\hline & & M1030509000 & 2001-01-25 06:33:00 \\
\hline & & M1030405000 & 2001-01-25 09:53:00 \\
\hline & & M1030608000 & 2001-09-28 13:50:00 \\
\hline & & M1030510000 & $2001-09-28$ 15:35:00 \\
\hline & & M1030406000 & 2001-09-28 17:15:00 \\
\hline & & M1030609000 & 2001-11-21 09:54:00 \\
\hline & & M1030511000 & 2001-11-21 11:39:00 \\
\hline & & M1030407000 & 2001-11-21 13:19:00 \\
\hline & & M1030610000 & 2002-02-17 07:27:00 \\
\hline & & M1030512000 & 2002-02-17 12:34:00 \\
\hline & & M1030408000 & 2002-02-17 17:43:00 \\
\hline & & M1030611000 & 2002-02-23 02:05:00 \\
\hline & & M1030513000 & 2002-02-23 06:43:00 \\
\hline & & M1030409000 & 2002-02-23 08:23:00 \\
\hline & & M1030612000 & $2002-02-2502: 17: 00$ \\
\hline & & M1030514000 & 2002-02-25 06:59:00 \\
\hline & & M1030613000 & 2002-12-03 21:00:00 \\
\hline & & M1030614000 & 2002-12-06 02:30:00 \\
\hline & & M1030515000 & 2002-12-06 05:16:00 \\
\hline & & M1052001000 & 2002-12-07 21:46:00 \\
\hline & & M1030615000 & 2002-12-08 22:36:00 \\
\hline & & M1030516000 & 2002-12-09 00:29:00 \\
\hline & & M1030412000 & 2002-12-09 03:53:00 \\
\hline & & M1030616000 & 2003-02-05 19:14:00 \\
\hline & & M1030517000 & 2003-02-05 21:07:00 \\
\hline & & M1030413000 & 2003-02-06 00:36:00 \\
\hline & & S4057604000 & $2003-11-2105: 21: 00$ \\
\hline & & M1030617000 & 2003-11-23 20:16:00 \\
\hline & & M1030519000 & 2004-01-25 21:31:00 \\
\hline & & M1030415000 & 2004-01-26 00:51:00 \\
\hline
\end{tabular}




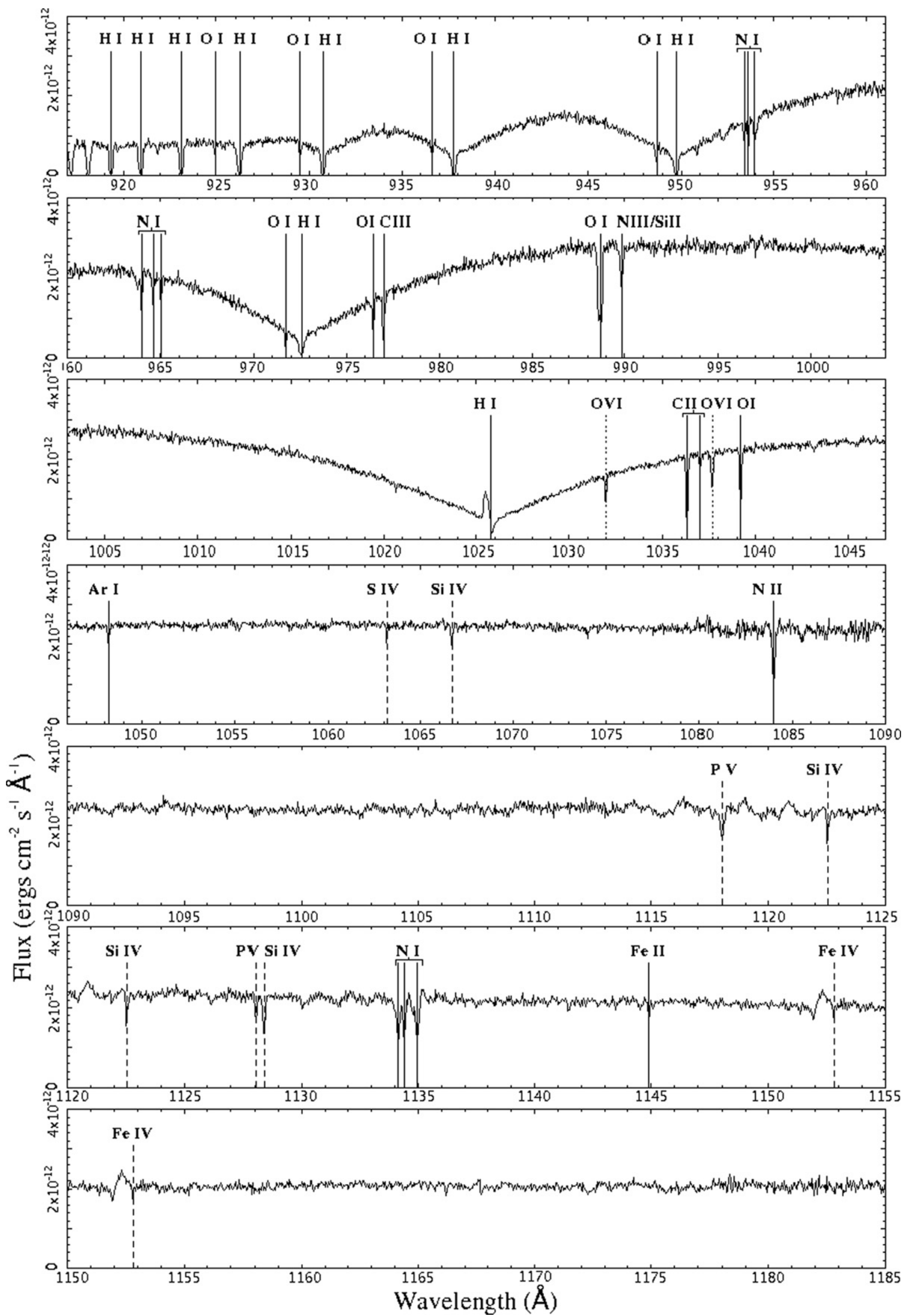

Figure 1. FUSE spectrum of the hot DA white dwarf, WD 0131-164 (GD 984), with the main photospheric and interstellar features labeled. The wavelengths of all these absorption lines can be obtained from Table 2. We note that the line identified as photospheric Si IV $1066.62 \AA$ in this plot may often be contaminated by interstellar Ar I, which occurs at the same wavelength. 


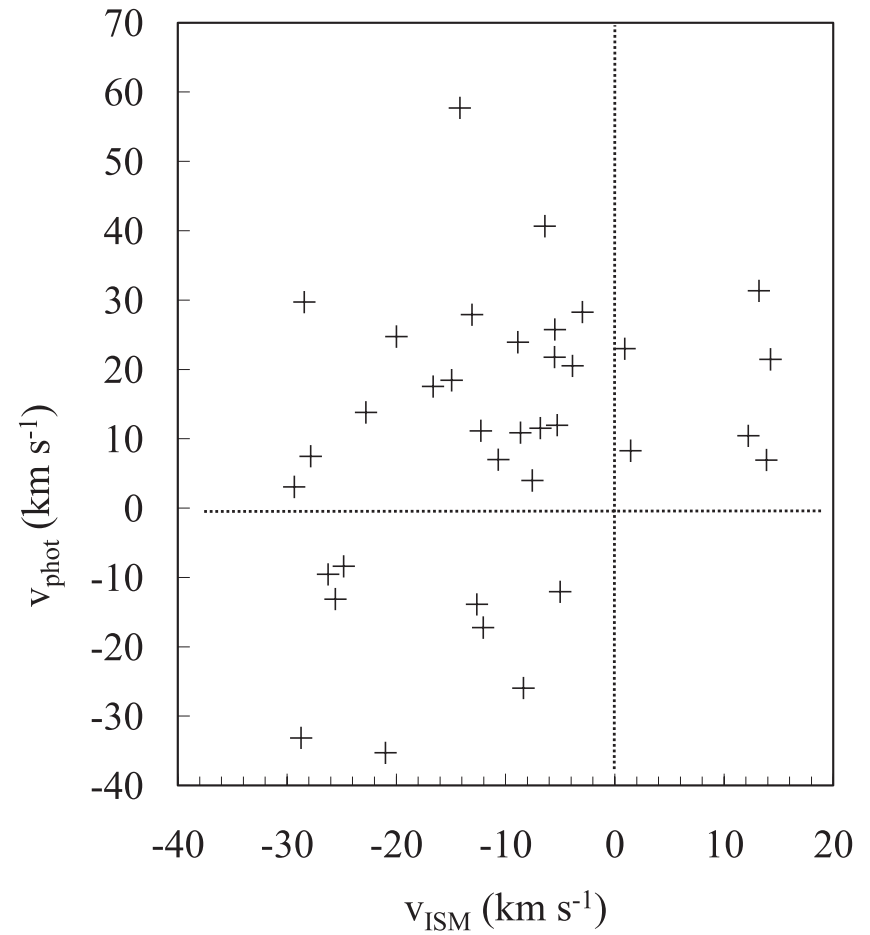

Figure 2. White dwarf heliospheric velocity ( $v_{\text {phot }}$ ) compared to the ISM velocity $\left(v_{\text {ISM }}\right)$ for the measurements listed in Table 3.

calculated velocity differences. Figure 2 compares the white dwarf photospheric heliocentric velocity with that measured for the ISM. There is no particular strong trend, but on average the photospheric velocities are offset by $\sim 15-20 \mathrm{~km} \mathrm{~s}^{-1}$, a value significantly lower than the $35.2 \pm 20.4 \mathrm{~km} \mathrm{~s}^{-1}$ obtained by Savage \& Lehner (2006). This is illustrated in Figure 3, showing the distribution of velocity differences $\left(v_{\mathrm{PHOT}}-v_{\text {ISM }}\right)$, which average $20.3 \pm 20.1 \mathrm{~km} \mathrm{~s}^{-1}$. Within the measurement uncertainties, this is close to the $20 \mathrm{~km} \mathrm{~s}^{-1}$ gravitational redshift of a typical 0.54 solar mass $\left(R=0.017 R_{\odot}\right)$ hot white dwarf star.

To monitor consistency, we have examined whether or not the velocities of individual lines vary systematically with wavelength and considered the scatter of the individual data points about the mean. Where we have reliable measurements of lines near $\mathrm{O}$ VI we have also compared these velocities with the computed means as a further consistency check. For all the stars in our study any discrepancies are well within the $\sim 15 \mathrm{~km} \mathrm{~s}^{-1}$ limit of the $R \sim 20,000$ FUSE resolving power.

Measurements of interstellar opacity made with a higher spectral resolution than is available with FUSE reveal that the interstellar lines of sight are often complex, with multiple components at different velocities (e.g., Sahu et al. 1999). Therefore, the interstellar lines observed in this work might well be blends of more than one component and the observed interstellar velocity the mean of these, weighted by the individual line strengths. The presence of unresolved blends may lead to increased scatter in the velocities we measure for individual ISM lines, as the weightings vary from line to line according to differing interstellar cloud conditions. However, in this work, we are mostly interested in defining the separation between the ISM and photospheric velocities. The only real issue is that we need to take into account these systematic uncertainties in the analysis.

Table 3 lists the photospheric (where measureable) and interstellar velocities for each star in the sample, determined using the approach outlined above. We do not give statistical errors for these measurements because they are usually very

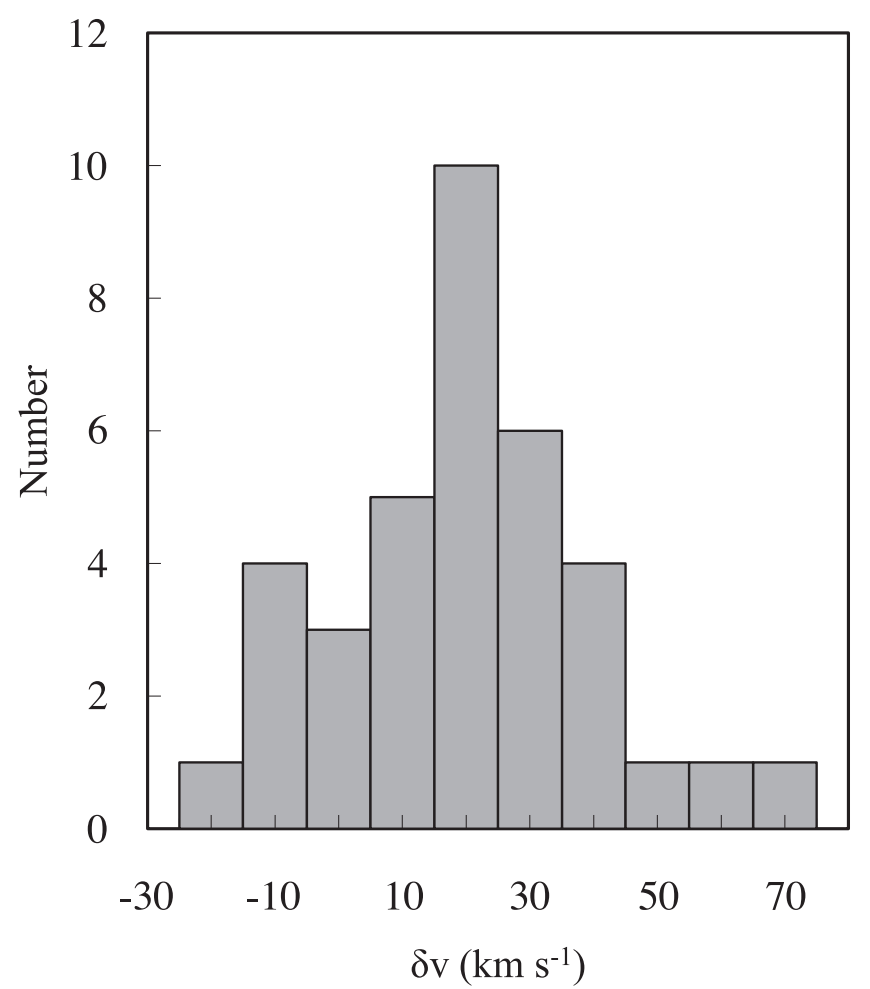

Figure 3. Number distribution of velocity differences $\left(v_{\mathrm{PHOT}}-v_{\mathrm{ISM}}\right)$. The distribution has a mean of $20.3 \mathrm{~km} \mathrm{~s}^{-1}$.

small compared to the systematic effects. The largest systematic issue is associated with the absolute velocity scale. However, any offsets apply equally to all our measurements and, as we are only interested in measuring the differences between the OVI, photospheric and ISM velocities, we can safely ignore this. In principle, the instrument resolving power gives an absolute limit $\left(15 \mathrm{~km} \mathrm{~s}^{-1}\right.$, corresponding to the nominal LWRS resolution of 20,000) to the determination of velocities, but making the assumption that the observed lines should be symmetrical we can usually achieve a factor of $\sim 3$ better using a centroid determination algorithm rather than merely measuring the velocity of the minimum flux pixel in the line. Therefore, we assign a general uncertainty of $5 \mathrm{~km} \mathrm{~s}^{-1}$ to all the present velocity measurements.

\subsection{Detection and Measurement of $O \mathrm{VI}$}

We have inspected every FUSE spectrum in our sample to look for evidence of the presence of O VI, taking into account the possible uncertainties in its location, depending on whether or not the two possible lines at $1031.912 \AA$ and $1037.613 \AA$ might be located at the interstellar or photospheric velocity (see Tables 3 and 4). We also widened the search to $\pm 0.2 \AA$ $\left(60 \mathrm{~km} \mathrm{~s}^{-1}\right)$ either side of the range to ensure we did not miss any $\mathrm{O}$ vi that might be associated with another velocity component. O VI $1031.912 \AA$ is detected in 41 objects in our sample, $\approx 50 \%$ of the objects studied, and the weaker $1037.613 \AA$ line is seen in 16 objects. The FUSE spectra in the vicinity of the O vi line detections are shown in Figure 4 for the 1031.912 $\AA$ line.

The $1037.613 \AA$ line is detected only when $1031.912 \AA$ is present, which is expected from the relative oscillator strengths. The issue of the significance of a detection of either $\mathrm{O}$ VI line is an important one, which obviously depends on the intrinsic line strength and the $\mathrm{S} / \mathrm{N}$ of the data. In many white dwarfs, the $\mathrm{O}$ VI lines are strong, and, therefore, obvious in the data, with their 
Table 3

Measured Photospheric and Interstellar Velocities $\left(\mathrm{km} \mathrm{s}^{-1}\right)$ for each White Dwarf where O VI 1031.912 $\mathrm{A}$ is Detected

\begin{tabular}{|c|c|c|c|c|c|c|c|c|c|c|c|}
\hline WD No. & $v_{\text {phot }}$ & $v_{\text {ism }}$ & $\delta v$ & $v_{\mathrm{O} \mathrm{VI}}$ & EW & $\sigma$ & $\mathrm{EW} / \sigma$ & $\left|v_{\mathrm{O} \text { vI }}-v_{\text {phot }}\right|$ & $\left|v_{\mathrm{O} v \mathrm{I}}-v_{\text {ism }}\right|$ & O vi? & $S \& L$ \\
\hline WD0001+433 & 7.0 & -10.7 & 17.7 & & $<23.4$ & & & & & ND & \\
\hline WD0004+330 & & -1.8 & & -11.0 & 6.1 & 1.2 & 5.0 & 11.0 & 9.2 & I & I \\
\hline WD0027-636 & 21.8 & -5.5 & 27.3 & 0.5 & 28.6 & 4.6 & 6.2 & 21.3 & 6.0 & $\mathrm{I}+\mathrm{P}$ & $\mathrm{P}$ \\
\hline WD0050-332 & 18.4 & -14.9 & 33.4 & 13.7 & 6.3 & 1.7 & 3.8 & 4.8 & 28.6 & $\mathrm{P}$ & $\mathrm{P}$ \\
\hline WD0106-358 & -12.1 & -5.0 & -7.1 & & $<15.3$ & & & & & $\mathrm{ND}$ & \\
\hline WD0131-164 & 10.9 & -8.6 & 19.5 & 19.5 & 43.2 & 3.0 & 14.5 & 8.6 & 28.1 & $\mathrm{P}$ & \\
\hline WD0147+674 & & -15.5 & & & $<26.1$ & & & & & ND & ND \\
\hline WD0226-615 & 25.8 & -5.5 & 31.2 & 9.3 & 16.4 & 4.5 & 3.6 & 16.5 & 14.8 & $\mathrm{P}$ & \\
\hline WD0229-481 & 23.0 & 0.9 & 22.1 & & $<12.0$ & & & & & ND & \\
\hline WD0232+035 & 4.0 & -7.6 & 11.5 & -3.5 & 8.4 & 1.4 & 5.9 & 7.5 & 4.1 & $\mathrm{P}$ & \\
\hline WD0235-125 & & 13.7 & & & $<20.6$ & & & & & ND & \\
\hline WD0236+498 & & -4.5 & & & $<19.8$ & & & & & ND & \\
\hline WD0252-055 & 10.4 & 12.2 & -1.8 & 16.2 & 23.9 & 6.7 & 3.6 & 5.8 & 4.0 & $\mathrm{P}$ & \\
\hline WD0320-539 & & 2.5 & & & $<15.8$ & & & & & ND & \\
\hline WD0325-857 & & -10.0 & & & $<5.3$ & & & & & ND & \\
\hline WD0346-011 & & 15.8 & & & $<5.7$ & & & & & ND & \\
\hline WD0353+284 & & 9.0 & & 25.6 & 69.9 & 11.3 & 6.2 & & 16.6 & IR & \\
\hline WD0354-368 & & 8.7 & & & $<44.9$ & & & & & ND & \\
\hline WD0455-282 & 57.7 & $-14.2 /-65.5$ & 71.9 & 59.3 & 11.3 & 2.3 & 5.0 & 1.6 & 73.4 & $\mathrm{IB}+\mathrm{P}$ & $\mathrm{I}+\mathrm{P}$ \\
\hline WD0501+524 & 21.5 & 14.3 & 7.2 & 20.4 & 4.8 & 0.5 & 10.7 & 1.1 & 6.1 & $\mathrm{P}$ & $\mathrm{P}$ \\
\hline WD0512+326 & 20.5 & -3.9 & 24.4 & 25.0 & 14.4 & 4.4 & 3.3 & 4.4 & 28.8 & $\mathrm{P}$ & \\
\hline WD0549+158 & 8.3 & 1.4 & 6.8 & & $<4.0$ & & & & & ND & ND \\
\hline WD0603-483 & & $17.5 /-34.6$ & & & $<16.5$ & & & & & ND & $I^{*}$ \\
\hline WD0621-376 & 31.3 & 13.2 & 18.1 & 34.3 & 4.7 & 1.0 & 4.5 & 3.0 & 21.1 & $\mathrm{P}$ & \\
\hline WD0659+130 & & -0.3 & & & $<26.3$ & & & & & ND & \\
\hline WD0715-704 & & -4.8 & & -11.3 & 11.6 & 3.1 & 3.7 & & 6.5 & I & I \\
\hline WD0802+413 & 6.9 & $13.9 / 61.4$ & -6.9 & & $<33.7$ & & & & & ND & ND \\
\hline WD0830-535 & & 11.1 & & -1.9 & 31.0 & 9.0 & 3.4 & & 13.0 & I & I \\
\hline WD0937+505 & & -6.5 & & & 40.7 & 10.7 & 3.8 & & & I & I \\
\hline WD1019-141 & 11.5 & -6.8 & 18.3 & & $<10.9$ & & & & & ND & $I^{*}$ \\
\hline WD1021+266 & 12.0 & -5.3 & 17.2 & 13.4 & 36.4 & 5.6 & 6.5 & 1.4 & 18.6 & $\mathrm{P}$ & \\
\hline WD1024+326 & & -14.0 & & & $<51.8$ & & & & & ND & \\
\hline WD1029+537 & 13.8 & -22.8 & 36.6 & 25.7 & 63.6 & 4.4 & 14.6 & 11.9 & 48.5 & $\mathrm{P}$ & \\
\hline WD1040+492 & -13.9 & $-12.6 /-47.9$ & -1.3 & 45.0 & 30.3 & 3.7 & 8.2 & 58.9 & 57.6 & IR & \\
\hline WD1041+580 & & -10.4 & & & $<14.6$ & & & & & ND & ND \\
\hline WD1057+719 & & -16.1 & & 19.8 & 26.3 & 2.9 & 9.1 & & 35.9 & IR & I \\
\hline WD1109-225 & & -4.9 & & -8.0 & 13.8 & 4.0 & 3.5 & & 3.1 & I & \\
\hline WD1234+481 & & -9.6 & & -6.4 & 10.4 & 3.0 & 3.5 & & 3.2 & $\mathrm{I}$ & I \\
\hline WD1254+223 & & -11.8 & & 17.4 & 15.1 & 5.0 & 3.0 & & 29.2 & IR & I \\
\hline WD1302+597 & & -6.7 & & & $<17.4$ & & & & & ND & \\
\hline WD1314+293 & & -10.1 & & 19.8 & 10.5 & 1.8 & 5.8 & & 29.8 & IR & I \\
\hline WD1337+701 & -17.2 & -12.1 & -5.2 & & $<113.4$ & & & & & ND & ND \\
\hline WD1342+442 & -13.1 & -25.6 & 12.5 & -12.2 & 72.3 & 8.0 & 9.1 & 0.9 & 13.3 & $\mathrm{P}$ & \\
\hline WD1440+753 & & -14.0 & & & $<11.9$ & & & & & ND & ND \\
\hline WD1528+487 & & -27.3 & & -34.3 & 23.8 & 4.1 & 5.8 & & 7.0 & I & I \\
\hline WD1550+130 & & $-9.9 / 43.8$ & & & $<23.0$ & & & & & ND & \\
\hline WD1603+432 & & -34.3 & & & $<16.1$ & & & & & ND & I \\
\hline WD1611-084 & -35.3 & $-21.0 / 40.6$ & -14.3 & -24.2 & 53.1 & 4.0 & 13.3 & 11.1 & 3.2 & $\mathrm{P}$ & \\
\hline WD1615-154 & & -68.3 & & & $<12.0$ & & & & & ND & ND \\
\hline WD1620+647 & & -31.7 & & & $<17.2$ & & & & & ND & ND \\
\hline WD1631+781 & & -11.8 & & -8.0 & 6.9 & 2.1 & 3.3 & & 3.8 & I & I \\
\hline WD1635+529 & & -22.5 & & & $<16.3$ & & & & & ND & \\
\hline WD1636+351 & & $-31.1 / 24.9$ & & & $<15.1$ & & & & & ND & $I^{*}$ \\
\hline WD1648+407 & & -28.4 & & -18.0 & 21.9 & 9.3 & 2.4 & & 10.4 & I & I \\
\hline WD1725+586 & & $-37.8 / 25.4$ & & 18.7 & 53.1 & 5.2 & 10.2 & & 56.5 & IR & \\
\hline WD1800+685 & & -29.0 & & -18.9 & 11.7 & 2.1 & 5.5 & & & I & I \\
\hline WD1819+580 & -8.4 & -24.8 & 16.4 & & $<13.7$ & & & & & ND & \\
\hline WD1844-223 & & -35.5 & & & $<7.4$ & & & & & ND & ND \\
\hline WD1845+683 & & -28.2 & & & $<32.2$ & & & & & ND & ND \\
\hline WD1917+509 & 3.1 & -29.3 & 32.4 & & $<12.5$ & & & & & ND & ND \\
\hline WD1921-566 & & -30.1 & & & $<22.2$ & & & & & ND & \\
\hline WD1942+499 & 7.5 & -27.8 & 35.3 & 6.4 & 15.7 & 3.1 & 5.1 & 1.1 & 34.2 & $\mathrm{P}$ & $P$ \\
\hline WD1950-432 & -26.0 & -8.4 & -17.6 & 0.3 & 17.5 & 5.0 & 3.5 & 26.3 & 8.7 & I & I \\
\hline WD2000-561 & -9.6 & -26.2 & 16.7 & -15.3 & 25.4 & 6.9 & 3.7 & 5.7 & 11.0 & $\mathrm{P}$ & $\mathrm{P}$ \\
\hline WD2004-605 & & -27.2 & & -18.0 & 9.4 & 2.8 & 3.4 & & 9.2 & I & I \\
\hline WD2011+398 & 11.1 & -12.3 & 23.4 & 13.9 & 62.6 & 3.5 & 18.0 & 2.7 & 26.1 & $\mathrm{P}$ & \\
\hline
\end{tabular}


Table 3

(Continued)

\begin{tabular}{|c|c|c|c|c|c|c|c|c|c|c|c|}
\hline WD No. & $v_{\text {phot }}$ & $v_{\text {ism }}$ & $\delta v$ & $v_{\mathrm{O} \text { VI }}$ & EW & $\sigma$ & $\mathrm{EW} / \sigma$ & $\varphi v_{\mathrm{O} \text { vI }}-v_{\mathrm{phot}} \varphi$ & $\varphi v_{\mathrm{O} \text { vI }}-v_{\mathrm{ism}} \varphi$ & O vi? & S\&L \\
\hline WD2014-575 & & -29.8 & & & $<29.0$ & & & & & ND & ND \\
\hline WD2020-425 & & -25.4 & & & $<42.0$ & & & & & ND & \\
\hline WD2111+498 & 24.7 & -20.0 & 44.7 & 13.1 & 20.1 & 4.2 & 4.8 & 11.7 & 33.1 & $\mathrm{P}$ & $\mathrm{P}$ \\
\hline WD2116+736 & & -17.6 & & -3.5 & 9.9 & 2.6 & 3.8 & & 14.1 & I & I \\
\hline WD2124+191 & & -19.7 & & & $<44.7$ & & & & & ND & \\
\hline WD2124-224 & 29.7 & -28.4 & 58.1 & -16.4 & 20.1 & 4.7 & 4.3 & 46.1 & 12.0 & I & I \\
\hline WD2146-433 & 27.9 & -13.1 & 41.0 & 10.4 & 20.3 & 4.5 & 4.6 & 17.5 & 23.5 & $\mathrm{P}$ & $\mathrm{P}$ \\
\hline WD2152-548 & & -3.9 & & -2.6 & 90.0 & 6.6 & 13.7 & & 1.3 & I & \\
\hline WD2211-495 & 23.9 & -8.9 & 32.8 & 18.0 & 15.4 & 1.2 & 13.4 & 5.9 & 26.9 & $\mathrm{P}$ & $\mathrm{P}$ \\
\hline WD2257-073 & & -10.2 & & 30.8 & 19.1 & 6.2 & 3.1 & & 41.0 & IR & \\
\hline WD2309+105 & -33.1 & -28.7 & -4.4 & & $<5.1$ & & & & & ND & ND \\
\hline WD2321-549 & 17.5 & -16.6 & 34.2 & -1.6 & 44.5 & 6.3 & 7.0 & 19.1 & 15.0 & $\mathrm{I}+\mathrm{P}$ & $\mathrm{P}$ \\
\hline WD2331-475 & 28.3 & -3.0 & 31.2 & 3.0 & 13.2 & 2.4 & 5.6 & 25.2 & 6.0 & $\mathrm{I}+\mathrm{P}$ & $\mathrm{P}$ \\
\hline WD2350-706 & 40.7 & -6.4 & 47.1 & 10.6 & 45.1 & 3.6 & 12.5 & 30.0 & 17.0 & $\mathrm{I}+\mathrm{P}$ & \\
\hline
\end{tabular}

Notes. Tabulated above are the difference between photospheric and ISM velocities; the wavelength, velocity, equivalent width (mA), and significance of the O VI $1031.912 \AA$ (EW/uncertainty in EW); absolute differences between the O VI and photospheric/ISM velocities; indication of the nature of the O VI absorption (P: photospheric, I: interstellar at $v_{\text {ism }}$, IR: interstellar redshifted w.r.t. $v_{\text {ISM }}$, IB: interstellar blueshifted w.r.t. $v_{\text {ISM }}$, ND: non-detection); nature of the O VI as determined by Savage \& Lehner (2006). The data are grouped by the nature of the O VI detected in these stars. Symbol “*” for these stars denotes the reason for the discrepancy between this work and Savage \& Lehner is their adoption of a $2 \sigma$ detection threshold.
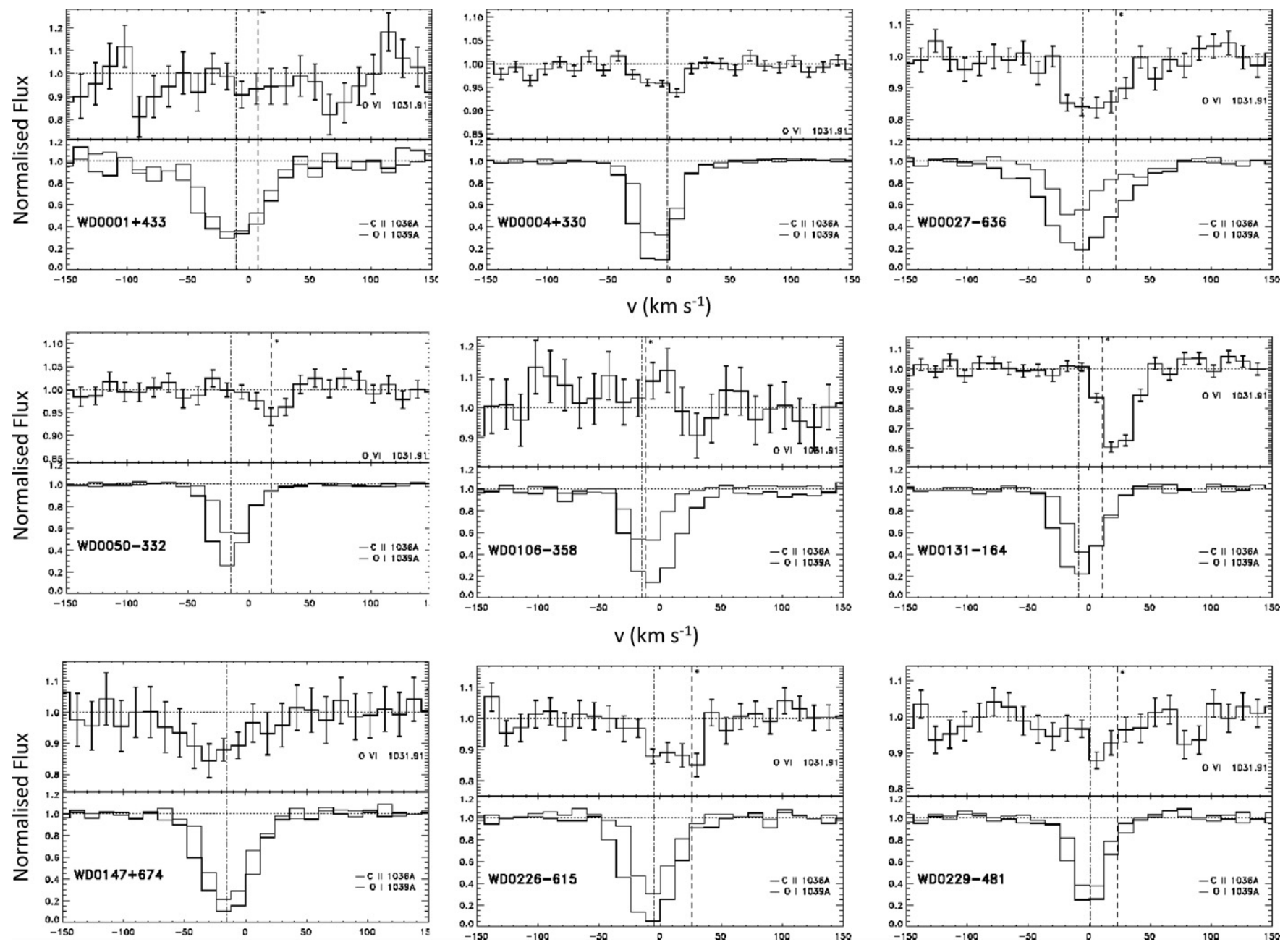

Figure 4. Normalized profiles vs. the heliocentric velocity of the local ISM C II and O I absorption lines (lower panel) compared to the O vI region. The vertical dashed line marks the photospheric velocity and the dash-dotted line the mean interstellar velocity for each star. We mark the statistical error bars in the top panel of each plot to illustrate the significance of the $\mathrm{O}$ VI detections we report but have left them out of the bottom panel for clarity.

location well determined. However, weaker lines, particularly those close to the noise limit, are not so readily identified and separating real O vi detections from "noise" features becomes problematic. We have measured the velocities of all detected $\mathrm{O}$ VI lines as described in Section 3.3. We also determined their equivalent widths (EWs) in the standard way, as the area of 

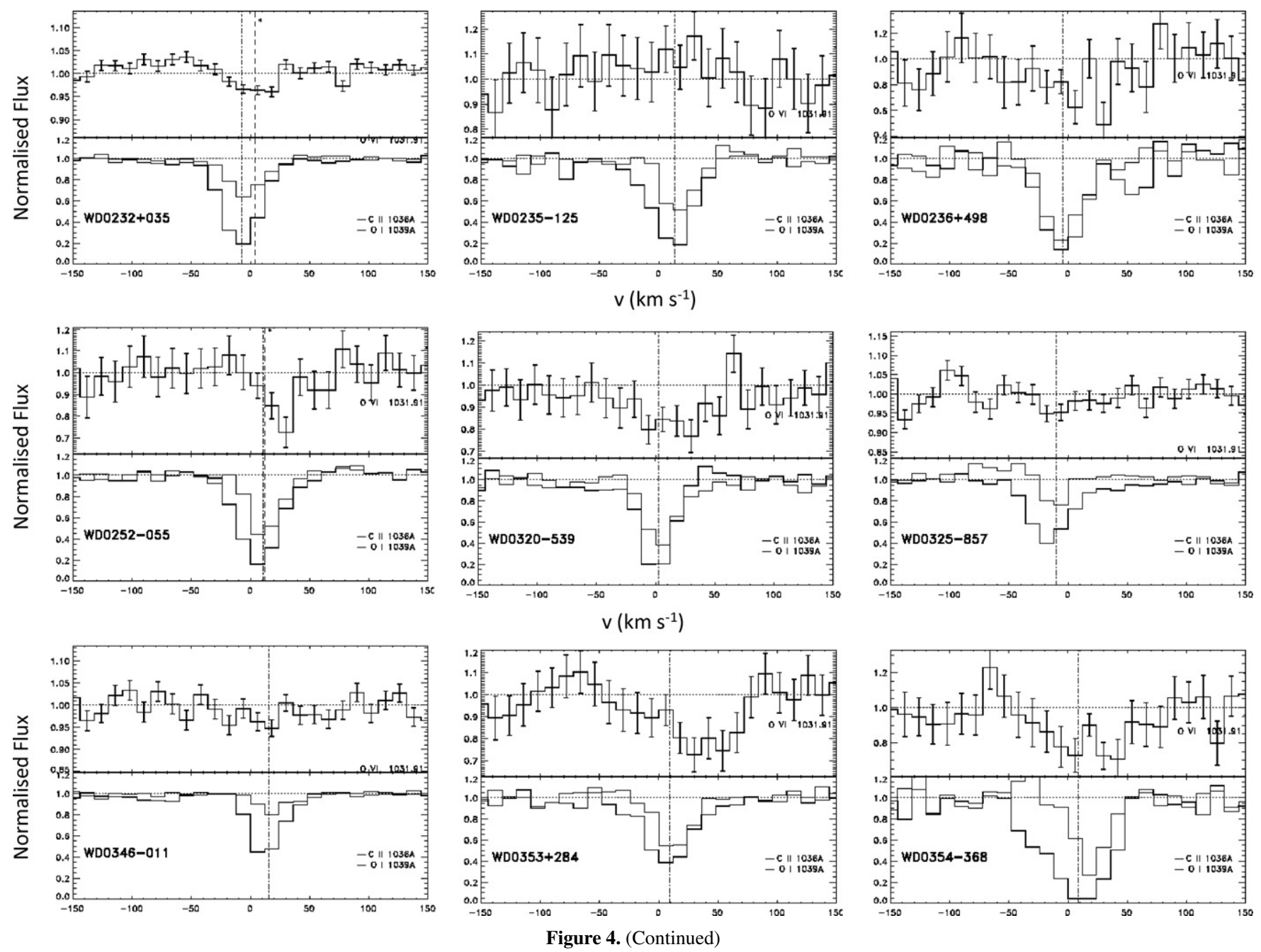

Table 4

Stars Where O vi $1037.613 \AA$ is Detected

\begin{tabular}{lcccrrrr}
\hline \hline WD No. & Star & $v_{\mathrm{O} \text { vi }(1031.912)}$ & O vi? & $v_{\text {O vi }(1037.613)}$ & EW & $\sigma$ & EW/ $\sigma$ \\
\hline WD0027-636 & REJ, EUVEJ & 0.49 & $\mathrm{I}+\mathrm{P}$ & 16.79 & 28.07 & 4.53 & 6.20 \\
WD0131-164 & GD984 & 19.46 & $\mathrm{P}$ & 18.59 & 50.29 & 2.47 & 20.36 \\
WD0252-055 & HD18131 & 16.24 & $\mathrm{P}$ & 27.26 & 28.47 & 4.88 & 5.83 \\
WD0455-282 & REJ, EUVEJ & 59.27 & $\mathrm{IB}+\mathrm{P}$ & -65.35 & 11.40 & 1.91 & 5.97 \\
WD0512+326 & HD33959C & 24.96 & $\mathrm{P}$ & 12.20 & 14.24 & 2.82 & 5.05 \\
WD0621-376 & REJ, EUVEJ & 34.34 & $\mathrm{P}$ & 25.15 & 2.90 & 0.85 & 3.41 \\
WD1021+266 & HD90052 & 13.36 & $\mathrm{P}$ & 12.27 & 32.08 & 4.74 & 6.77 \\
WD1029+537 & REJ, EUVEJ & 25.68 & $\mathrm{P}$ & 24.09 & 50.47 & 3.46 & 14.59 \\
WD1342+442 & PG & -12.23 & $\mathrm{P}$ & -20.14 & 69.07 & 6.96 & 9.92 \\
WD1528+487 & REJ, EUVEJ & -34.34 & $\mathrm{I}$ & -32.52 & 18.78 & 4.00 & 4.70 \\
WD1611-084 & REJ, EUVEJ & -24.20 & $\mathrm{P}$ & -26.24 & 44.96 & 3.93 & 11.44 \\
WD1615-154 & G153-41 & 46.72 & $\mathrm{ND}$ & 20.17 & 7.67 & 3.00 & 2.56 \\
WD1725+586 & LB335 & 18.70 & $\mathrm{I}$ & 18.09 & 45.75 & 4.94 & 9.26 \\
WD2011+398 & REJ, EUVEJ & 13.89 & $\mathrm{P}$ & 11.18 & 44.76 & 2.78 & 16.10 \\
WD2124-224 & REJ, EUVEJ & -16.39 & $\mathrm{I}$ & 11.25 & 5.04 & 2.93 & 1.72 \\
WD2152-548 & REJ, EUVEJ & -2.61 & $\mathrm{I}$ & -8.99 & 55.42 & 4.78 & 11.59 \\
WD2211-495 & REJ, EUVEJ & 18.04 & $\mathrm{P}$ & 18.20 & 13.71 & 1.16 & 11.82 \\
\hline
\end{tabular}

the line falling below the nominal continuum flux level. The associated uncertainty in the EW is estimated from the size of adjacent noise features in the spectrum, which allows us to assign a measure of significance for each "detection". Where there is no clear detection, we have determined a $3 \sigma$ upper limit to the line strength from the typical size of noise in the vicinity of the $\mathrm{O}$ VI location.
Table 3 includes all O vi 1031.912 Å measurements, giving the measured wavelength, calculated radial velocity of the line, the EW, its uncertainty, and its significance (the EW divided by the uncertainty). However, where only an upper limit to the $\mathrm{O}$ VI line strength is obtained, we only give that $\mathrm{EW}$ as the other parameters are not relevant. Of the "detected" lines, the measured significance ranges from a maximum $\sim 18 \sigma$ down to 

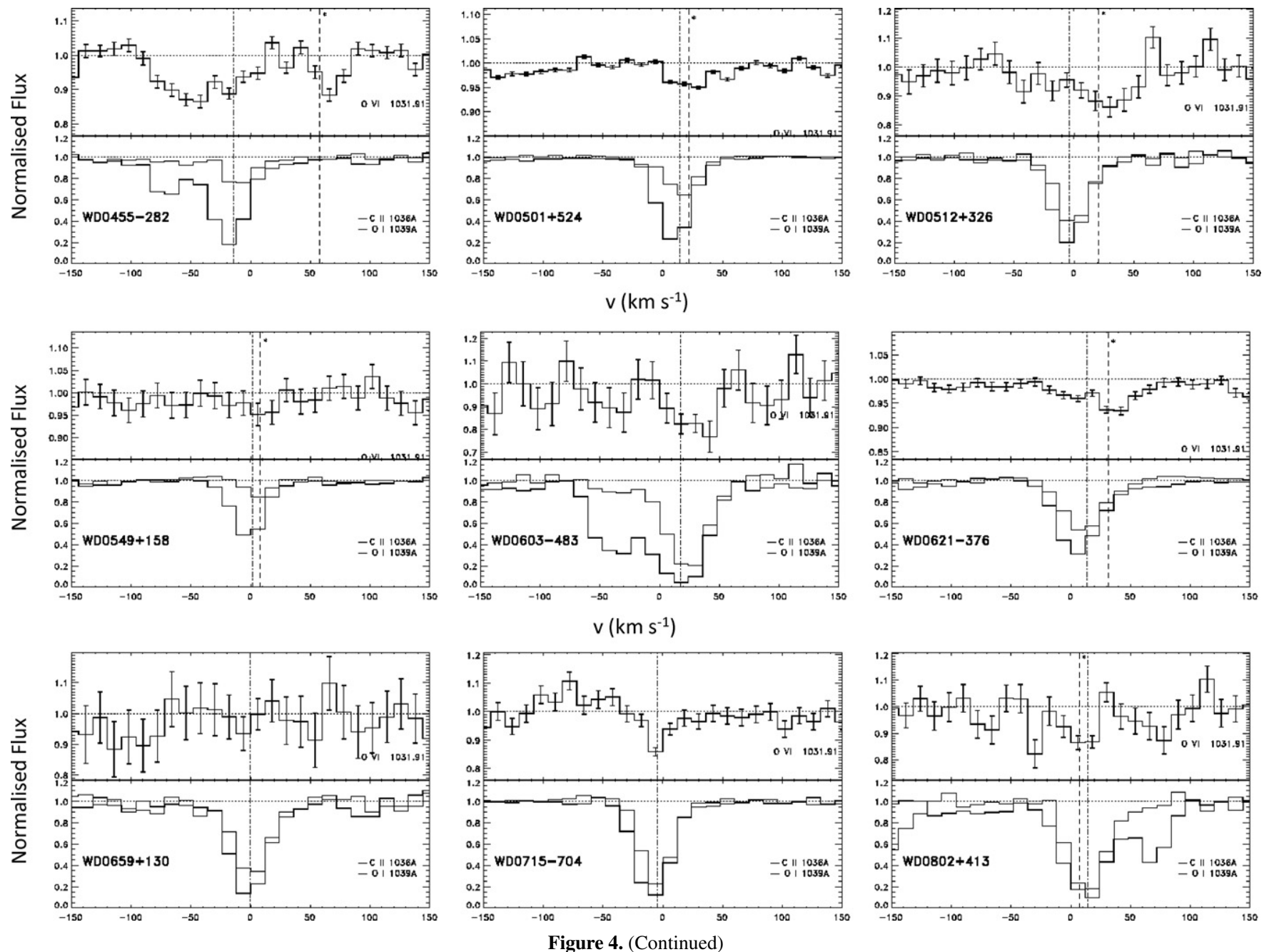

our $3 \sigma$ threshold. Any detection below $3 \sigma$ is not considered to be significant and is excluded from further analysis and discussion, apart from as an upper limit to the amount of $\mathrm{O}$ VI present. Similar data for the O VI $1037.613 \AA$ detections are given in Table 4. All the upper limits for detection of O VI $1031.912 \AA$ are listed in Table 3 but we provide no upper limits for the nondetections of the $1037.613 \AA$ line because these far outnumber the reliable detections and, since they are calculated from the general noise level in the spectrum, will be the same as the values listed for the $1031.912 \AA$ line.

A number of stars in the sample show evidence for multiple stellar components. Where these are clearly detected we note the velocity of the additional component in Table 3 . There is no clear evidence of any secondary $\mathrm{O}$ VI component associated with any of these weaker features. However, in WD0445-282, the interstellar O VI absorption is broad and spans the weaker feature. There is also a hint in Figure 4 that there might be a secondary $\mathrm{O}$ VI feature in WD1725+586, but it does not formally exceed our applied $3 \sigma$ threshold. We discuss the particular properties of some individual stars and lines of sight in the Appendix.

\section{DISCUSSION}

\subsection{Determination of the Origin of the $O \mathrm{VI}$}

As described earlier in this paper, there is strong evidence from other observations that relatively high-ionization stages are seen in white dwarf photospheres at temperatures below those limits expected from stellar atmosphere calculations, due to stratification of material in the stellar envelopes. Therefore, when any metals are present in the atmosphere of a white dwarf we are unable to rely on the ionization stage and stellar temperature as a means of discriminating between a photospheric and interstellar origin for the O VI. The only completely reliable method we can then use is to compare the $\mathrm{O}$ vi radial velocity with the values obtained for the photosphere and ISM in each star.

How well we can discriminate between a photospheric and interstellar origin depends on the separation of the two velocity components and the uncertainty in the velocity measurements. In Section 3.3, we estimated that the systematic and limiting uncertainty in the velocity measurements was $\sim 5 \mathrm{~km} \mathrm{~s}^{-1}$. Hence, the photospheric and interstellar components must be at least separated by this amount for us to be able to discriminate. However, this is a somewhat naïve and likely underestimate of the magnitude of velocity difference that can be excluded with high confidence. First, we are looking at velocity differences and, therefore, the combined uncertainty of two velocity measurements needs to be taken into account. Furthermore, the velocity measurement error adopted corresponds to a $\sim 1 \sigma$ value. Therefore, there is a $68 \%$ probability that the true velocity lies within the quoted range of uncertainty. If we are to claim that a particular O vi line resides at either photospheric or interstellar velocity, we need to be able to rule out an association with the other component at high confidence. Adopting a $2 \sigma$ 

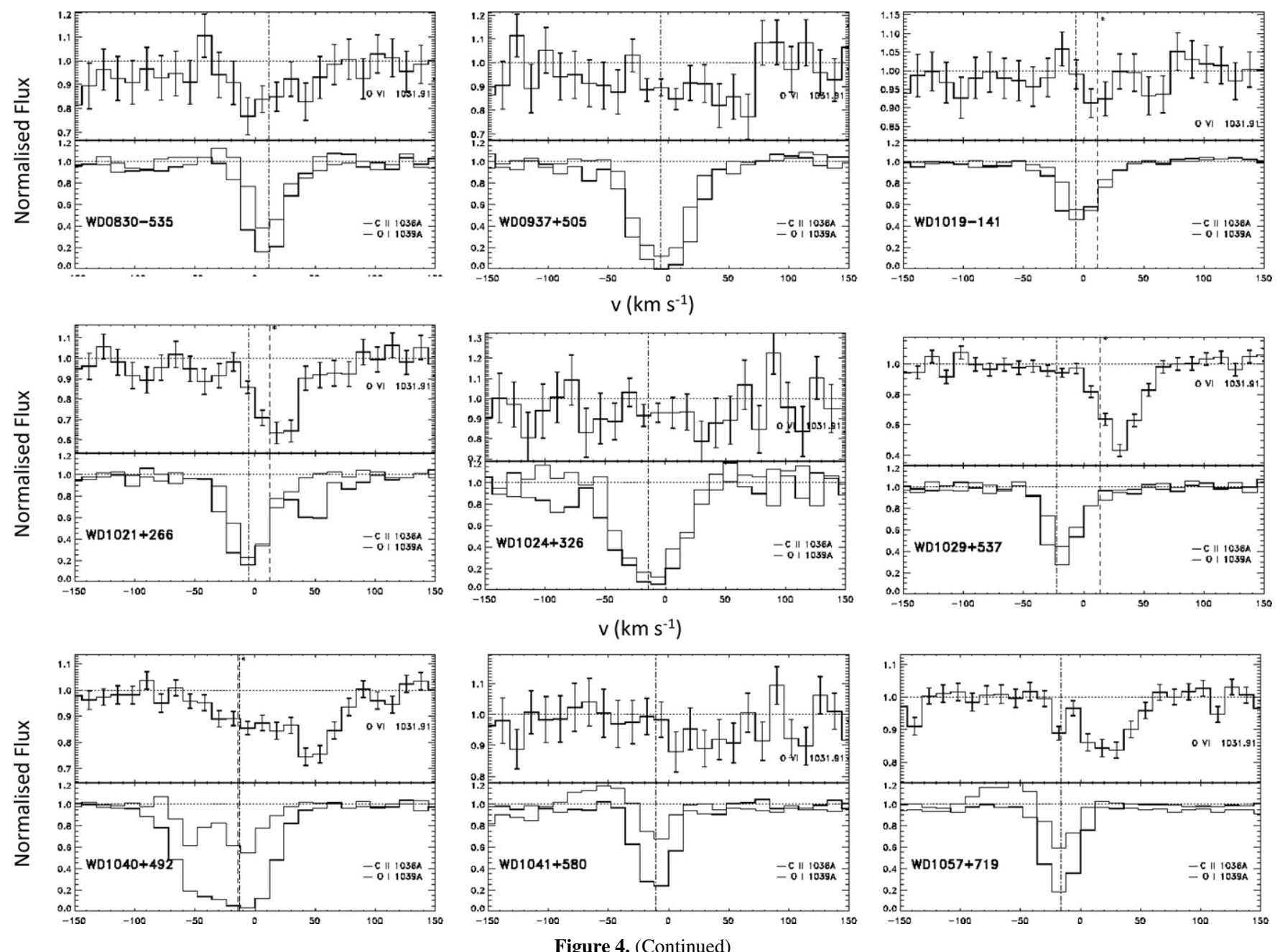

$\left(10 \mathrm{~km} \mathrm{~s}^{-1}\right)$ uncertainty yields a $95 \%$ confidence limit. For the purposes of deciding the nature of the $\mathrm{O}$ VI components we look for a $14 \mathrm{~km} \mathrm{~s}^{-1}\left(\sqrt{2} \times 10 \mathrm{~km} \mathrm{~s}^{-1}\right)$ minimum separation to rule out an association.

\subsubsection{Detection of Photospheric $O$ VI}

As discussed in Section 1, O vi can be present in white dwarf photospheres and is not necessarily restricted to hightemperature objects. The detections are only designated photospheric if the detected line resides close to the measured stellar velocity and is well separated from the location of the expected interstellar component. Good examples are WD0131-164 or WD2211-495. Slightly problematic are objects such as WD1029+537. In this example, the core of the O vI line is 1-2 wavelength bins redward of the photospheric velocity. However, this remains within the overall measurement uncertainties and so this is also classified as a likely photospheric detection. In any case, there is sufficient ambiguity to rule it out as interstellar in the context of this analysis. In many stars, we have a clear detection of $\mathrm{O}$ VI but the velocity difference between the ISM and photosphere is insufficiently large to make a clear statement about the nature of the detection. For example, in WD0501+524, the two components are virtually coincident, while in WD1611-084 they are separated by $\sim 15 \mathrm{~km} \mathrm{~s}^{-1}$, but still too small, when compared to the width of the line and the accuracy of the velocity measurements to assign an origin.
In the hottest white dwarfs, it is most likely that the O vI is photospheric in origin. Below $\sim 45,000-50,000 \mathrm{~K}$ this is not necessarily the case, but since we know of several examples (WD0050-332 is one) where highly ionized metals are present at lower temperatures we cannot be definitive about the origin of the $\mathrm{O} v \mathrm{vI}$.

\subsubsection{Detection of Interstellar $O \mathrm{VI}$}

Any detection recorded at a velocity other than the photospheric value we attribute to interstellar material. In 43 out of 80 white dwarfs a thorough search of the FUSE spectrum reveals no detectable photospheric material. If the $\mathrm{O}$ VI were photospheric, we would expect to see evidence of some other species. Therefore, we conclude that any $\mathrm{O}$ vi present is most likely to be interstellar in these stars. For most detections, the velocity of $\mathrm{O}$ VI was coincident, within measurement uncertainties, with that of the cooler ISM, as determined from neutral or singly ionized species. However, in several cases the O vI velocity was redshifted with respect to the ISM, as illustrated by WD1057+719 and WD1040+492. In one star, WD0455-282, the $\mathrm{O} \mathrm{VI}$ is blueshifted.

\subsubsection{Detection of Both Interstellar and Photospheric $O$ VI}

Interestingly, there is also a clearly resolved photospheric component in the spectrum of this WD0455-282. There may also be a weak component at the ISM velocity, but this cannot 

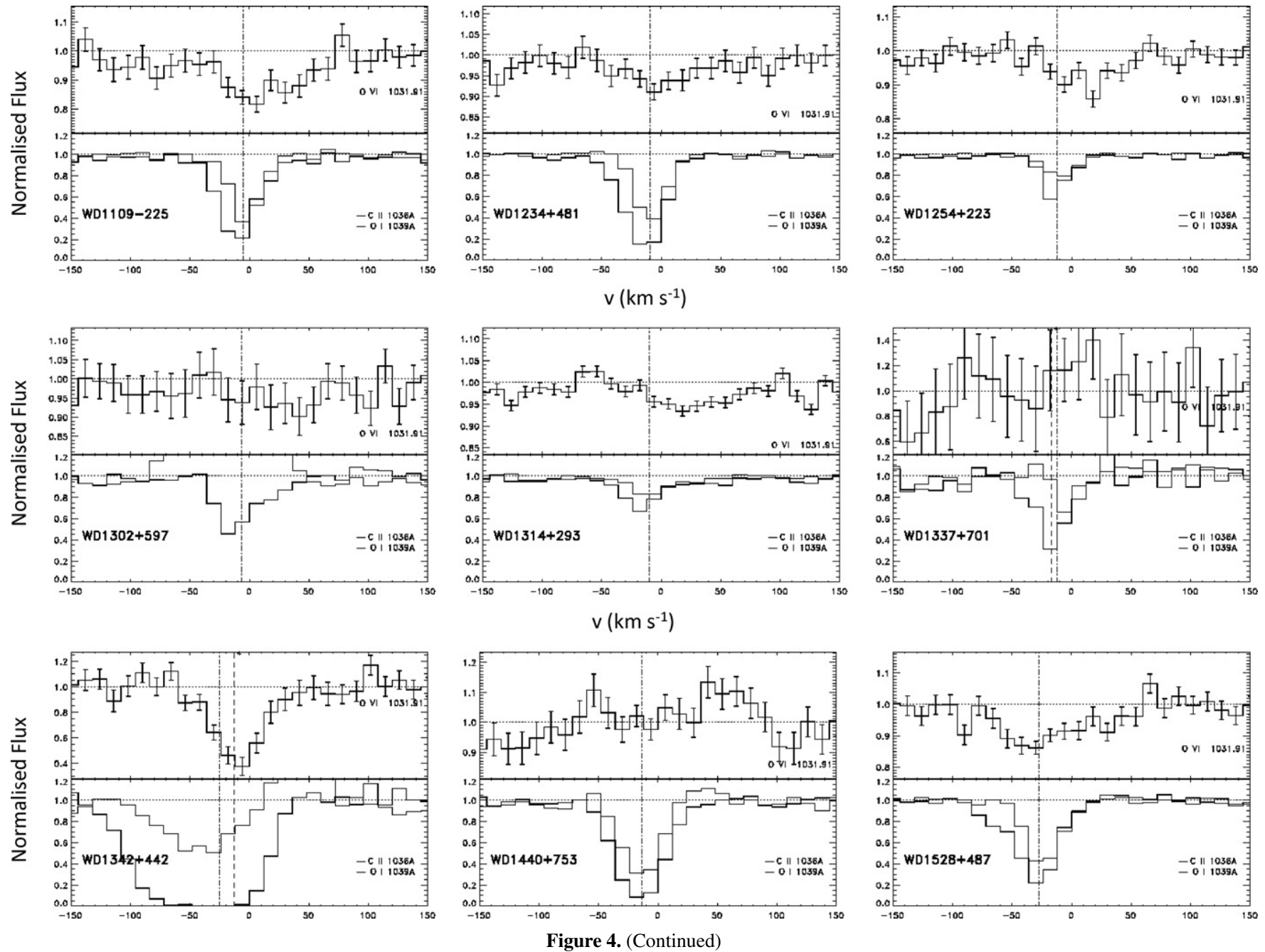

be resolved from the broad blueshifted feature. There are three other objects where both interstellar and photospheric components appear to be present. However, the separation of the ISM and photospheric velocity components is not large enough to completely resolve these. For example, WD2321-549 exhibits a broad feature which appears to have two minima aligned with each velocity component. If there were no interstellar component or if it was much weaker than the photospheric contribution, the features would be strongly skewed toward the photospheric velocity. Hence, this is a clear indication that this is a blend of two lines. Three other stars, WD0027-636, WD2331-475, and WD2350-706, show similarly broad lines, which are good evidence for two blended components, but with less distinct separation. For these stars we record the full strength of the line from both contributions in Table 3 but take into account the photospheric contamination, which is approximately $50 \%$ of the total in these cases, by fitting an additional Gaussian profile in estimating the interstellar column and volume densities in Table 5. As a result, there will be some additional systematic uncertainty in the measurements. We have not included this in the error estimates but indicate the increased uncertainty in Tables 5 and 6.

Taking all the above into account we label the most likely origin of the $\mathrm{O}$ VI in Table 3 as follows: definitely or possibly photospheric $(\mathrm{P})$, interstellar at $v_{\text {ISM }}(\mathrm{I})$, interstellar redshifted relative to $v_{\text {ISM }}$ (IR), interstellar blueshifted relative to $v_{\text {ISM }}$ (IB), not detected (ND). The interpretation of Savage \& Lehner
(2006) is also shown for comparison with our results. It is important to note that when we used the designation "P," we are indicating that the star contains other photospheric components which make it likely that there is a significant photospheric contribution to the $\mathrm{O}$ vi feature. However, this does not definitively exclude the possibility of an interstellar component being present, hidden by or blended with a photospheric contribution when the velocity separation is below the instrument resolution. In four stars, (WD0027-636, WD2321-549, WD2331-475, and WD2350-706), we see broad O vi components which we interpret as blended photospheric and interstellar lines and designate "I+P," even though not fully resolved. For these stars, the photospheric and interstellar components are severely blended and for our estimate of the interstellar contribution, we make the assumption that the photospheric and interstellar components are roughly equal in strength. The result is of course very uncertain and that is why we do not estimate any errors and use the symbol " " to indicate the high level of uncertainty in Tables 5 and 6 . In other examples, where the photospheric feature is dominant the presence of any ISM component is much more ambiguous and we are not able to carry out the same analysis.

\subsection{Comparison with Other Studies}

As discussed extensively already in this paper, there have been two previous studies of interstellar O vi using white dwarfs as background sources, by Oegerle et al. (2005) and Savage \& 

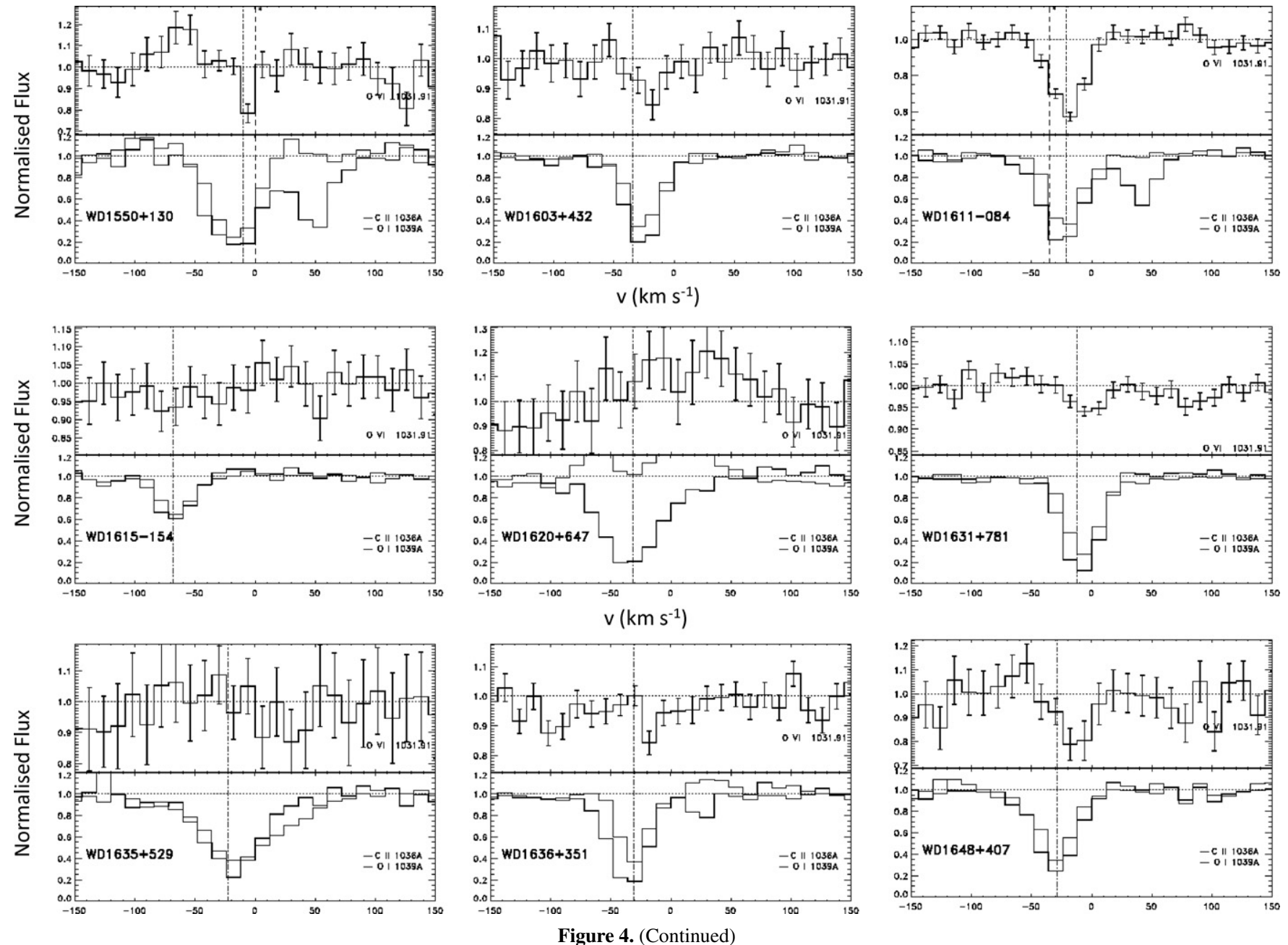

Lehner (2006). Essentially, the Oegerle et al. sample is a subset of that of Savage \& Lehner. Therefore, in this section we will just compare our results with the more recent paper. There is considerable agreement between the analysis we have conducted here and that of Savage \& Lehner. We have 26 detections of interstellar material, compared to their 21 for the stars that are in common with this study. Since we apply a higher $(3 \sigma$, cf. $2 \sigma$ ) detection threshold a few detections reported by Savage \& Lehner (WD0603-483, WD1019-141, WD1603+432, and WD1636+351) are not included in our list, although we do obtain similar EW and significance in each case. There are two stars in the Savage and Lehner sample (WD0027-636 and WD2321-549) where they designated the O VI as photospheric but which appear to have a blended ISM component (also noted by them) and where we have estimated the strength of the ISM component.

Despite the fact that the sample of DA white dwarfs studied in the work is almost a factor of 2 larger than that of Savage \& Lehner, we have detected interstellar O VI in only eight additional lines of sight a $\sim 25 \%$ increase when counting just the $3 \sigma$ and above detections, indicating a rather lower "success rate" in detecting interstellar O VI. Three of these (WD0027-636, WD2331-475, and WD2350-706) are also apparent blends of ISM and photospheric contributions. Hence, this may be partly due to Savage \& Lehner's a priori exclusion from their analyses previously known stars where the $\mathrm{O}$ VI is thought to be contaminated or where the $\mathrm{S} / \mathrm{N}$ is not adequate, which we did consider here.

\section{3. $O$ vi Column and Volume Densities}

We have reliable temperature and gravity estimates for all the white dwarfs in our sample, obtained from either Balmer or Lyman line analyses, as listed in Table 1. Coupling this information with visual magnitude and/or flux data allows us to estimate a distance to each object. This process depends on the assumption that the standard mass-radius relation for a typical DA white dwarf is correct (see Barstow et al. 2005, for further discussion on this topic) but with that, and the additional proviso that there are no hidden companions, yields distances accurate to a few percent (Holberg et al. 2008) and which we have listed in Table 1. Consequently, we can examine both the distribution of column and volume densities for the $\mathrm{O}$ vi detections and upper limits. The upper limits obtained are listed in Table 3 and span a range of EWs from $4.0 \mathrm{~m} \AA$ for the very highest $\mathrm{S} / \mathrm{N}$ spectra to $113 \mathrm{~m} \AA$, where the data have poor $\mathrm{S} / \mathrm{N}$. Since all the detected $\mathrm{O}$ VI lines are relatively weak, it is reasonable to assume that they are not saturated. Therefore, column densities have been calculated using the standard linear curve-of-growth method for unsaturated lines:

$$
N=W_{\lambda} /\left(8.85 \times 10^{-21} f \lambda^{2}\right)
$$

where $N$ is the column density $\left(\mathrm{cm}^{-2}\right), W_{\lambda}$ is the EW of the observed line/upper limit ( $\mathrm{m} \AA$ ), and $f$ is the oscillator strength of the line $(0.133$ for O VI at $\lambda=1031.93 \AA)$. The average volume density along the line of sight $\left(n, \mathrm{~cm}^{-3}\right)$ is then calculated simply by dividing by the distance to the star. The results of these 

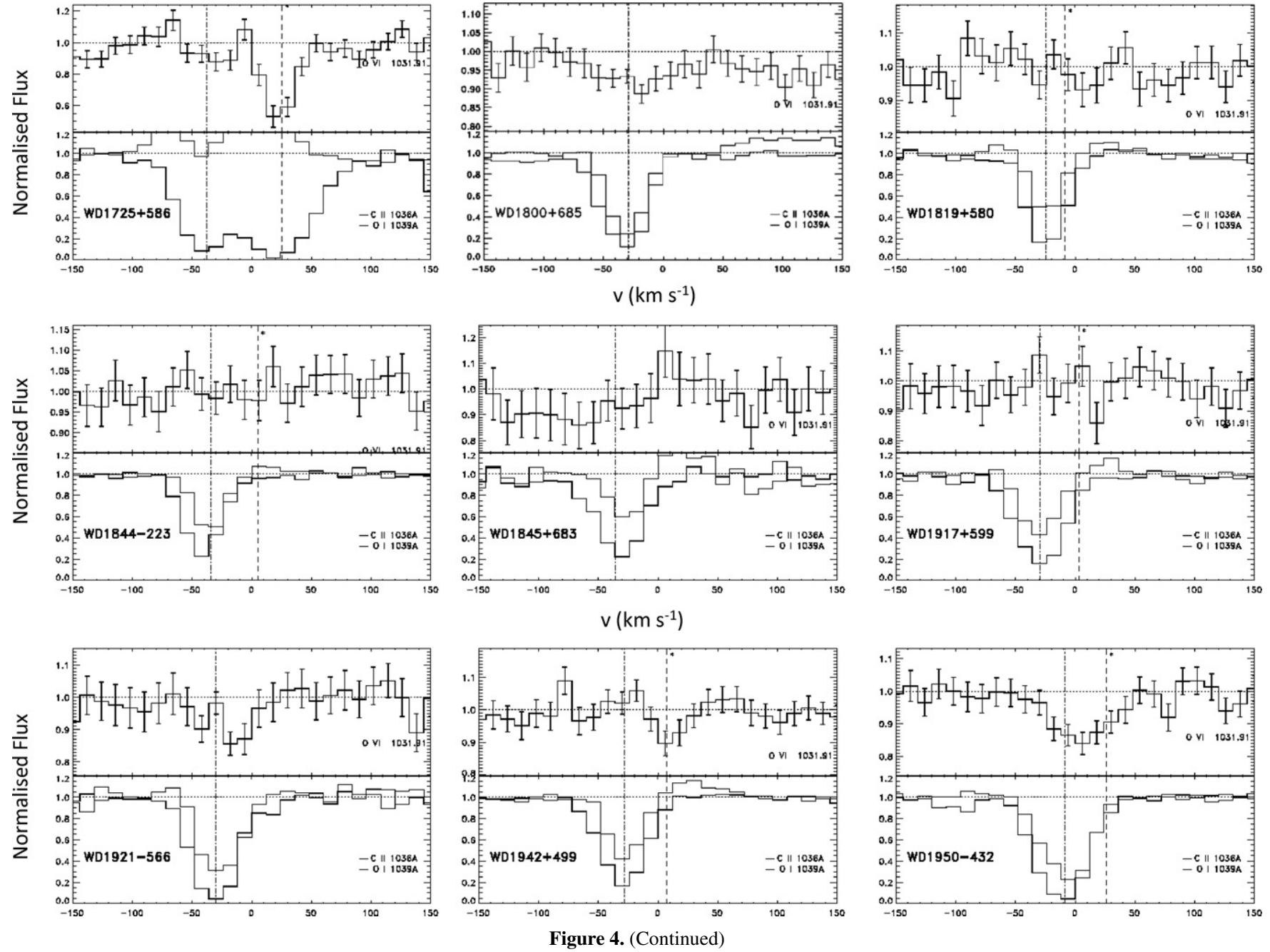

calculations are summarized in Table 5 and in Figures 5 and 6, which show column and volume density as functions of stellar distance, respectively. The black diamonds and error bars are the measured values while the gray diamonds are the upper limits. For a number of stars with the highest $\mathrm{S} / \mathrm{N}$ the implied densities determined from the upper limits have lower values than the actual detections. Hence, these limits provide tight constraints on the amount of hot gas present along those particular lines of sight.

One possible model for the LB is that it is full of hot X-ray emitting gas. If this material were uniformly distributed, we would expect to see $\mathrm{O}$ VI detections exclusively from transition temperature gas at the interface with cooler material, near the bubble boundary. Closer examination of Figure 5 shows that several of the well-constrained upper limits are obtained for stars that are closer to the Sun than the main group of detections. However, at least half the upper limits are at similar distances to stars with interstellar detections. Indeed, one non-detection (WD0229-481) is the most distant object in this group. This is a strong indication that the distribution of material around the Sun is far from uniform and that interfaces are not seen in all directions.

We already know from earlier studies of the LISM, culminating in the work of Lallement et al. (2003), that the boundary of the LB is not equidistant from the Sun in all directions. Nor is it a simple shape. So perhaps it is not surprising that there is significant variance in the measured column densities and upper limits. For sources within the LB, we might expect the conversion to volume density to produce a more coherent picture. In fact, as can be seen in Figure 6, this is not so, with very low column densities being recorded over some of the larger distances. Again, the picture is one of extreme patchiness, with volume densities varying by an order of magnitude between stars at similar distances.

\subsection{The Spatial Distribution of the Local O VI Absorption}

Previous authors have already described the distribution of O VI absorption within 200 pc of the Sun as being "patchy" (Oegerle et al. 2005; Savage \& Lehner 2006). If O vi forms at the evaporative interfaces between cool clouds and the surrounding hot million degree (soft X-ray emitting) LB gas, then the presence of tangled or tangential magnetic fields could quench thermal conduction over a large portion of a cloud's surface and thus O vi would only be formed in "patches" (Cox \& Helenius 2003). However, Welsh \& Lallement (2008) have suggested that many sight lines along which local interstellar O vI absorption $(d<100 \mathrm{pc})$ and $\mathrm{O}$ VI emission have been detected seem to preferentially occur at high galactic latitudes, in regions that also possess high levels of SXRB emission. To test this possible spatial correlation claim further with our new data, in Figure 7 we show the galactic distribution of the white dwarf sight lines within $120 \mathrm{pc}$ together with B star sight lines (also within $120 \mathrm{pc}$ ) listed by Welsh \& Lallement (2008) and Bowen et al. (2008) toward which O vi absorption has been detected with 

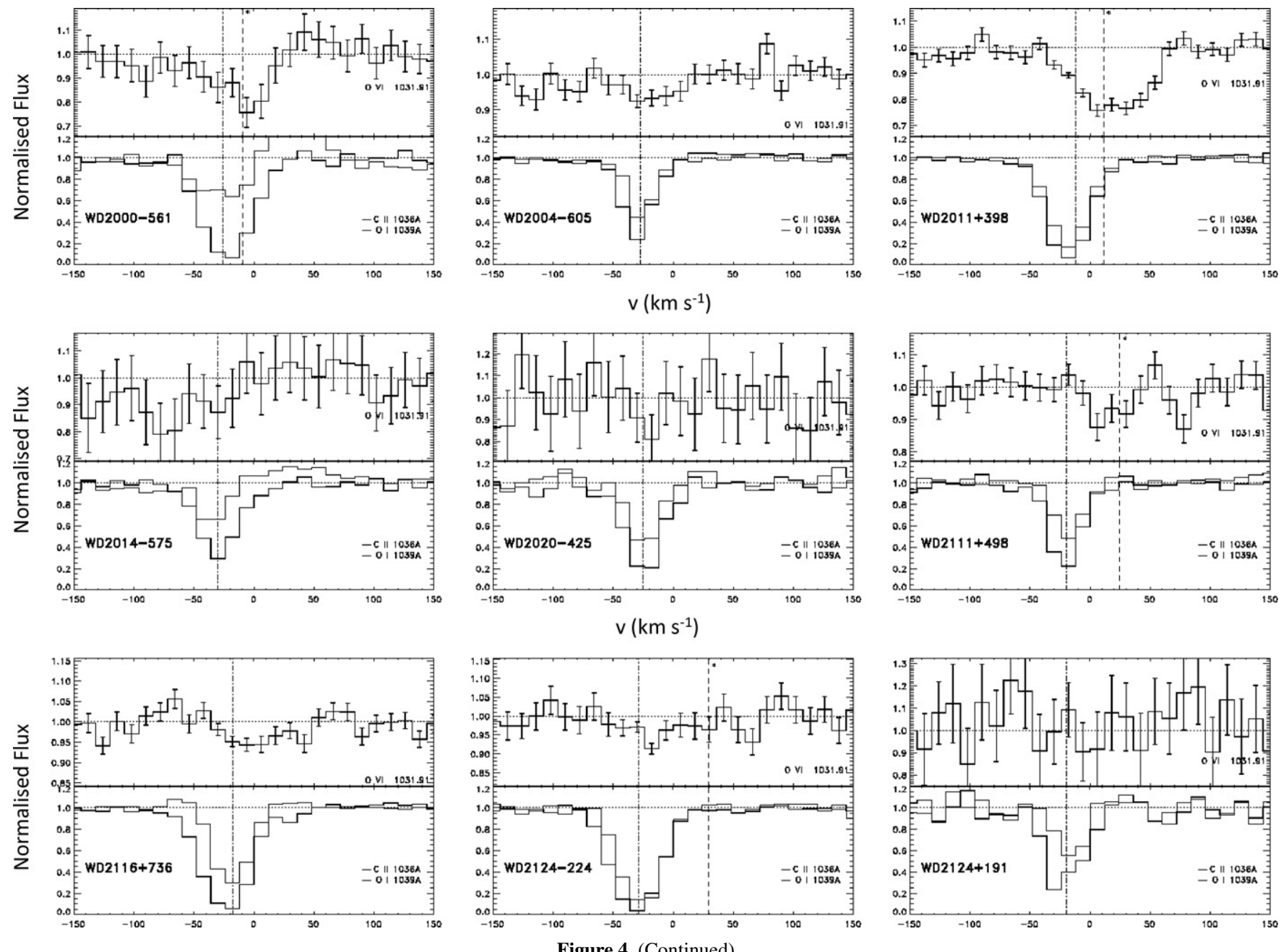

confidence, together with those sight lines (within the same distances) where we have measured only an upper limit to the possible O vi absorption strength. In Figure 7, these O vi data are superposed on a plot of the galactic distribution of the SXRB as mapped with the ROSAT satellite by Snowden et al. (1998). For the purposes of this discussion we define areas of "enhanced SXRB emission" as those with a flux of $>650$ R 12 counts.

An initial inspection of the distribution of $\mathrm{O}$ VI detections presented in Figure 7 seems to confirm the previous claim of a "patchy" distribution. However, a deeper inspection of the data reveals the following. In total, Figure 7 contains 49 sight lines, 15 of which have confirmed detections of interstellar O VI absorption. Of the 15 detections only two sight lines are not coincident with directions where the SXRB signal is enhanced over the lowest levels of SXRB emission. This seems to imply that whenever $\mathrm{O} V I$ is detected it tends to be in regions of enhanced SXRB emission. However, there are also 26 sight lines located in regions of enhanced SXRB emission where O VI was not detected. Therefore, the presence of enhanced SXRB along a sight line alone is insufficient to explain why $\mathrm{O}$ VI is preferentially detected.

In addition, of the six $\mathrm{O}$ vi detections located at mid-plane latitudes $\left(-30<b<+30^{\circ}\right)$, all are placed beyond $80 \mathrm{pc}$ (i.e., located presumably beyond the neutral boundary to the LB). This suggests that at mid-plane latitudes there is a deficiency of $\mathrm{O}$ VI bearing gas within the confines of the local cavity. The remaining nine $\mathrm{O}$ VI detections (regardless of distance) are all located at high galactic latitudes $\left(|b|<-30^{\circ}\right.$ and $\left.|b|>30^{\circ}\right)$, all located in regions of enhanced SXRB flux. However, there are also 15 non-detections at high latitudes that are located in regions of enhanced SXRB flux. Therefore, the location of a sight line at high latitudes is in itself insufficient to explain why $\mathrm{O}$ VI is sometimes detected and sometimes not.

We note that recent calculations on the spatial distribution of the SWCX by Koutroumpa et al (2009) have suggested that the LB may contain far cooler and more tenuous gas at low latitudes, with the SXRB at high latitudes arising from an external hot and highly ionized gas located in the overlying galactic halo (Welsh \& Shelton 2009). Although Figure 7 only very tentatively supports a scenario in which interstellar O vi could be formed in a transition region between hot (halo) gas and lower temperature (LB) gas at high latitudes, the presence of the (albeit few) O vI detections at low latitudes requires an alternative explanation. This is now provided by our interpretation of Figure 8.

Figure 8 indicates the location of a selection of the stars listed in Table 3 and shows a number of slices through the local ISM based on the three-dimensional spatial distribution of neutral gas presented by Lallement et al. (2003), but updated by Welsh et al. (2010) with more recent information. On each of the projections shown, we have plotted the positions of detections of interstellar $\mathrm{O} v \mathrm{v}$ along with the most sensitive non-detections. Each map shows the neutral gas density in a pseudo-grayscale, white representing low density and black high density, perpendicular to the galactic plane. All distances are in parsecs 

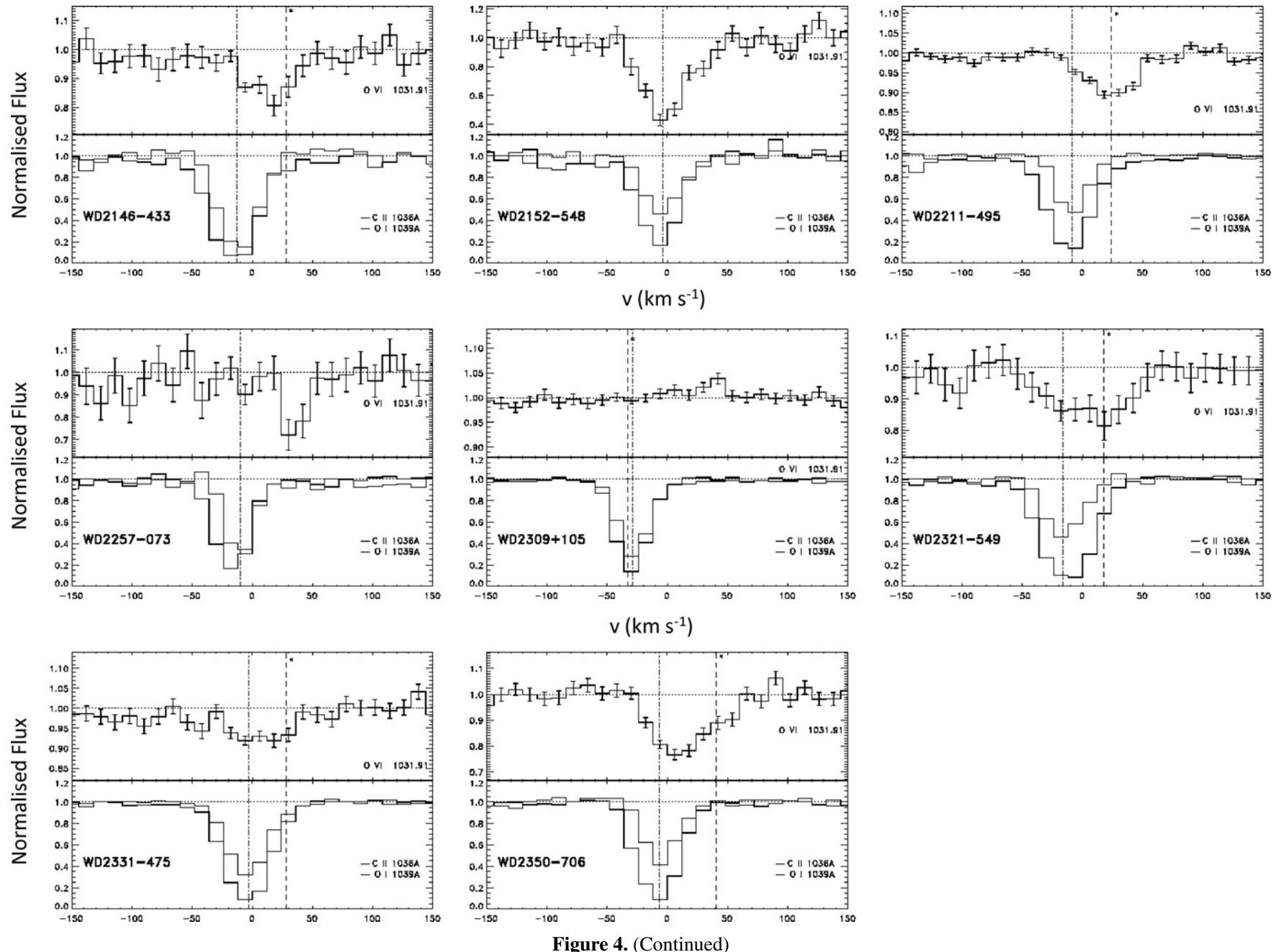

Figure 4. (Continued)

and the longitude of each slice marked along with the north and south galactic poles. All stars are marked with the log of their $\mathrm{O}$ vi column density and a number, for clarity, with the key to this labeling given in Table 6. Stars marked with filled circles correspond to ISM detections while the open circles represent non-detections. One star (21), a non-detection of O VI, lies at a distance above $200 \mathrm{pc}$ and is outside the boundary of the maps. Its direction is indicated by the arrow and distance noted on the plot. It is important to note that the density distributions of Lallement et al. (2003) have been obtained by inversion of a limited number of sight lines. As a consequence, their spatial resolution is coarse (of the order of 25 parsecs) and there may be clouds with a smaller extent that have not been detected and included in these maps. However, within these limitations, they give an idea of the ISM distribution along the lines of sight to the white dwarfs in this study.

Figure 8 shows that for the five white dwarf sight lines located well within the inner regions of the Local cavity (i.e., white regions in Figure 8), we find only two O vi detections (toward targets 31 and 32), the nearest detection being toward WD2004-605 (target 32) at a distance of 58 pc. Again, this supports the notion that there is a deficiency of $\mathrm{O}$ VI-bearing gas within the local cavity. Figure 8 also shows that the majority of white dwarfs yielding interstellar detections of $\mathrm{O}$ VI are mostly located close to or beyond the neutral boundary to the local cavity in regions of higher gas density (darker shaded regions in Figure 8). Some O vi detections lie at high galactic latitude, including most of the redshifted components (as noted by Savage \& Lehner 2006).

We note that in order to produce $\mathrm{O}$ VI an interface between hot (million Kelvin) and cooler gas is required. Since the LB is known to possess numerous partially ionized cloudlets (Redfield \& Linsky 2008), it would appear that there are sufficient interfaces for $\mathrm{O}$ vi to form if the hot million degree gas is ubiquitous throughout the LB. However, our observations indicate a distinctly patchy spatial distribution for O vI. Thus, it would appear that the hot gas density within the LB may be generally too low to produce detectable $\mathrm{O}$ VI at most cloud interfaces, but that there are regions, probably at the boundaries of the cavity and at interstellar cloud interfaces where there is sufficiently dense (or compressed) hot material. We note that the evolution of a supernova remnant (SNR) located within an existing low-density interstellar environment (i.e., a similar situation to that of the LB cavity) has been modeled for emission from high ions (Shelton 1998, 1999). These models predict that a middle-aged SNR should have a low density and hot (million degree) gas in the interior and a zone of O vi-rich gas at the boundary. With the passage of time the million degree gas in the cavity interior cools sufficiently for its X-ray emission to fade significantly (perhaps to the level calculated by Koutroumpa et al. 2009). The SNR cooling model predicts that within a timescale of only $\sim 2.5 \times 10^{5}$ years the transition temperature 


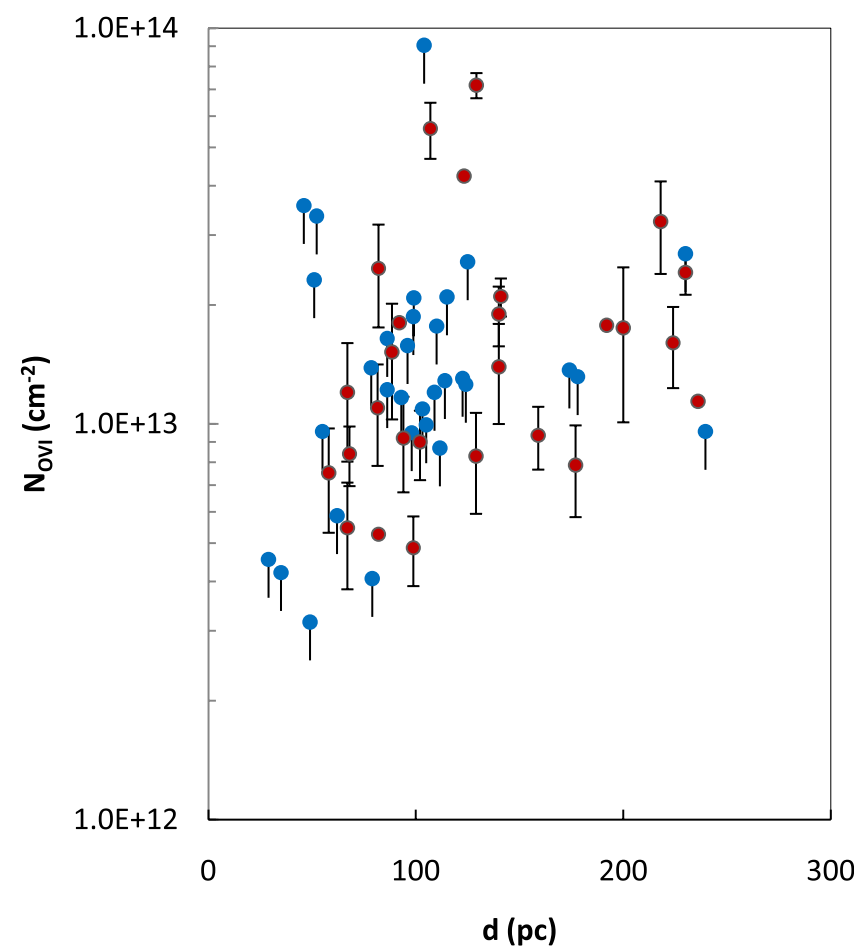

Figure 5. O vi column density as a function of stellar distance (pc). The filled red circles and error bars are measured values, while the filled blue circles and downward bar are $3 \sigma$ upper limits. For the four stars that have blended ISM and photospheric $\mathrm{O}$ VI components, the error bars are not shown because of the larger and unquantifiable uncertainty in the measurement.

(A color version of this figure is available in the online journal.)

ions such as $\mathrm{N} \mathrm{v}$ and $\mathrm{O}$ vI are to be mainly found at the periphery of the cooling SNR bubble and the rapidly cooling central gas is overionized and may be out of equilibrium. By $10^{6}$ years (i.e., less than the age of the LB), the inner cavity regions will have cooled sufficiently that the level of X-ray emission is negligible, while appreciable amounts of $\mathrm{O}$ VI ions should lie within the transition temperature gas at the bubble's edge. The data for our mid-plane detections of O VI could fit such a scenario.

Finally, we note the presence of a particularly interesting pair of stars residing in the region of the $l=340^{\circ}$ slice, where a detection and non-detection are spatially very close together (nos. 32 and 33; WD2004-605, and WD2014-575), lying on very similar lines of sight with the latter being just $5 \mathrm{pc}$ more distance. The detection (WD2004-605) is weak, $\sim 4 \sigma$, so it is conceivable that this is a threshold effect where the additional distance to this object allows the O vi column density to exceed the detectable level. However, there are other non-detections at similar or greater distances within the LB (e.g., stars 1, 8, 17, 19, 35, 36) along other lines of sight.

\subsection{Toward an Understanding of the $O$ VI-SXRB Connection}

The data presented in Figures 7 and 8 suggest that there is a general deficiency of $\mathrm{O}$ vi gas within the local cavity. In addition, for local detections of $\mathrm{O}$ VI near the galactic plane a sight line must either cross or be located at the edge of a neutral cloud at the LB boundary. Plots of the $E$-vector polarization of starlight by dust in the ISM presented by Mathewson \& Ford (1970) show that, for sight lines $<50 \mathrm{pc}$, optical polarization is minimal in all galactic directions with no preferential polarization vector angle. However, the polarization for stars in the 50-100 pc range clearly exhibits large features in the longitude range

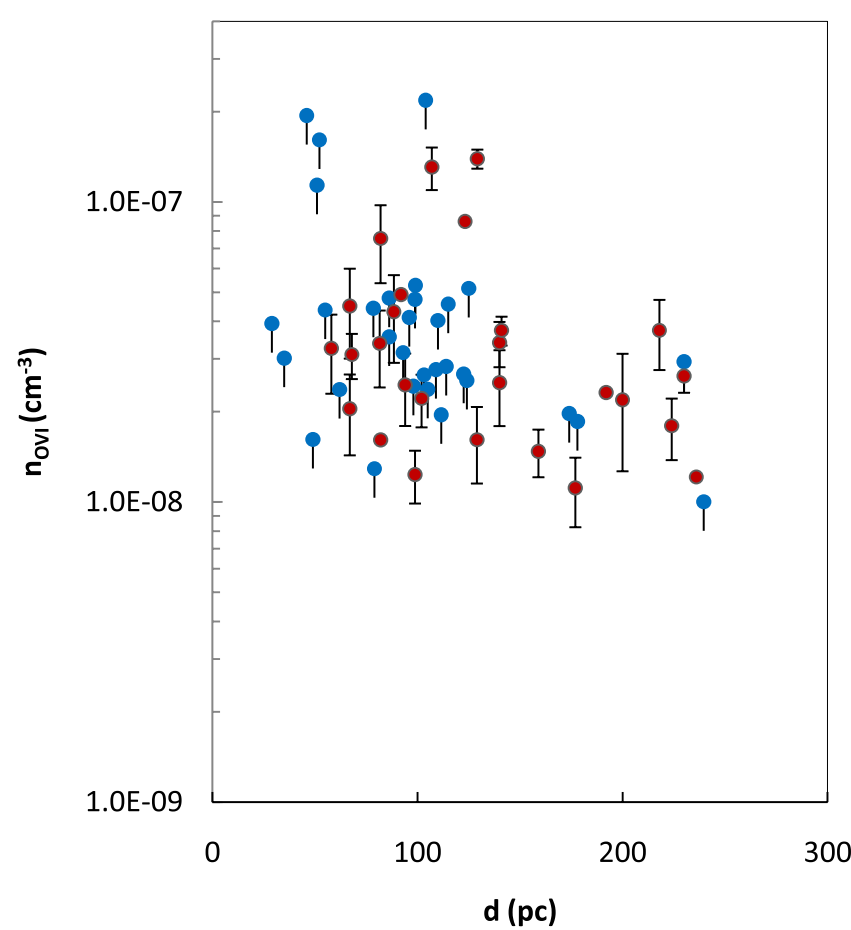

Figure 6. O vi volume density as a function of stellar distance (pc). The filled red circles and error bars are measured values, while the filled blue circles and downward bar are $3 \sigma$ upper limits. For the four stars that have blended ISM and photospheric $\mathrm{O}$ VI components, the error bars are not shown because of the larger and unquantifiable uncertainty in the measurement.

(A color version of this figure is available in the online journal.)

$l=0^{\circ}-60^{\circ}$ reaching up from the galactic plane to high positive latitudes. This polarization vector strengthens for stars in the 100-200 pc distance range, and a similar (but weaker) feature also emerges toward negative galactic latitudes. This feature in the optical polarization data (which results from the alignment of interstellar dust and gas due to a magnetic field) is also mimicked in maps of the galactic distribution of polarized $408 \mathrm{MHz}$ radio emission (Wolleben 2007). The feature is directly associated with the North Polar Spur, which is the brightest filament of the extensive Loop I superbubble (Heiles 1998). It is widely believed that the Loop I superbubble was formed by stellar winds and supernovae events caused by the Sco-Cen OB association of young stars. Its emission has been mapped in both X-rays and radio emission, and Egger (1998) argues that Loop I is caused by a shock from a recent supernova that has heated the inner wall of the Sco-Cen supershell. It is highly likely that this event also could have caused a blow-out from the galactic plane in the form of a galactic fountain.

In the various panels of Figure 8, we have shown the spatial distribution of cold and neutral gas in the meridian plane of the galaxy within $200 \mathrm{pc}$, as traced by NaI absorption measurements (Lallement et al. 2003) for comparison with our O VI absorption measurements. These maps clearly show the Local Chimney feature (Welsh et al. 1999) from several different viewpoints. This is an extension of the local cavity reaching toward high and low galactic latitudes. It was first noted by Welsh et al. (1999) that the angle of the Local Chimney is tilted toward the directions where the SXRB emission is greatest in both galactic hemispheres. The absorption characteristics of gas lying close to the axis of the Chimney have been probed by Crawford et al. (2002) and Welsh et al. (2004). These observations show that interstellar gas with velocities in the -20 to $-60 \mathrm{~km} \mathrm{~s}^{-1}$ range appears to be falling down the Chimney toward the galactic disk. 
Table 5

Measured Equivalent Widths, Errors, and Column and Volume Densities of Interstellar O vi for all the Detections Reported in this Paper

\begin{tabular}{|c|c|c|c|c|c|c|c|}
\hline WD No. & $d(\mathrm{pc})$ & EW & $\sigma$ & $N_{\mathrm{O} \text { VI }}\left(\mathrm{cm}^{-2}\right)$ & Error & $n_{\mathrm{O} \text { vI }}\left(\mathrm{cm}^{-3}\right)$ & Error \\
\hline WD0001+433 & 99 & 23.4 & & $<1.9 \mathrm{E}+13$ & & $<4.7 \mathrm{E}-08$ & \\
\hline WD0004+330 & 99 & 6.1 & 1.2 & $4.9 \mathrm{E}+12$ & $9.7 \mathrm{E}+11$ & $1.2 \mathrm{E}-08$ & $2.5 \mathrm{E}-09$ \\
\hline WD0027-636 & 236 & 14.3 & 4.6 & $\sim 1.1 \mathrm{E}+13$ & & $\sim 1.2 \mathrm{E}-08$ & \\
\hline WD0106-358 & 86 & 15.3 & & $<1.2 \mathrm{E}+13$ & & $<3.6 \mathrm{E}-08$ & \\
\hline WD0147+674 & 99 & 26.1 & & $<2.1 \mathrm{E}+13$ & & $<5.3 \mathrm{E}-08$ & \\
\hline WD0229-481 & 240 & 12.0 & & $<9.6 \mathrm{E}+12$ & & $<1.0 \mathrm{E}-08$ & \\
\hline WD0235-125 & 86 & 20.6 & & $<1.6 \mathrm{E}+13$ & & $<4.8 \mathrm{E}-08$ & \\
\hline WD0236+498 & 96 & 19.8 & & $<1.6 \mathrm{E}+13$ & & $<4.1 \mathrm{E}-08$ & \\
\hline WD0320-539 & 124 & 15.8 & & $<1.3 \mathrm{E}+13$ & & $<2.5 \mathrm{E}-08$ & \\
\hline WD0325-857 & 35 & 5.3 & & $<4.2 \mathrm{E}+12$ & & $<3.0 \mathrm{E}-08$ & \\
\hline WD0346-011 & 29 & 5.7 & & $<4.5 \mathrm{E}+12$ & & $<3.9 \mathrm{E}-08$ & \\
\hline WD0353+284 & 107 & 69.9 & 11.3 & $5.6 \mathrm{E}+13$ & $9 \mathrm{E}+12$ & $1.3 \mathrm{E}-07$ & $2.1 \mathrm{E}-08$ \\
\hline WD0354-368 & 400 & 44.9 & & $<3.6 \mathrm{E}+13$ & & $<2.2 \mathrm{E}-08$ & \\
\hline WD0455-282 & 102 & 11.3 & 2.3 & $9.0 \mathrm{E}+12$ & $1.8 \mathrm{E}+12$ & $2.2 \mathrm{E}-08$ & $4.4 \mathrm{E}-09$ \\
\hline WD0549+158 & 49 & 4.0 & & $<3.2 \mathrm{E}+12$ & & $<1.6 \mathrm{E}-08$ & \\
\hline WD0603-483 & 178 & 16.5 & & $<1.3 \mathrm{E}+13$ & & $<1.9 \mathrm{E}-08$ & \\
\hline WD0659+130 & 115 & 26.3 & & $<2.1 \mathrm{E}+13$ & & $<4.6 \mathrm{E}-08$ & \\
\hline WD0715-704 & 94 & 11.6 & 3.1 & $9.2 \mathrm{E}+12$ & $2.5 \mathrm{E}+12$ & $2.5 \mathrm{E}-08$ & $6.7 \mathrm{E}-09$ \\
\hline WD0802+413 & 230 & 33.7 & & $<2.7 \mathrm{E}+13$ & & $<2.9 \mathrm{E}-08$ & \\
\hline WD0830-535 & 82 & 31.0 & 9.0 & $2.5 \mathrm{E}+13$ & $7.2 \mathrm{E}+12$ & $7.6 \mathrm{E}-08$ & $2.2 \mathrm{E}-08$ \\
\hline WD0937+505 & 218 & 40.7 & 10.7 & $3.2 \mathrm{E}+13$ & $8.5 \mathrm{E}+12$ & $3.7 \mathrm{E}-08$ & $9.8 \mathrm{E}-09$ \\
\hline WD1019-141 & 112 & 10.9 & & $<8.7 \mathrm{E}+12$ & & $<2.0 \mathrm{E}-08$ & \\
\hline WD1024+326 & 400 & 51.8 & & $<4.1 \mathrm{E}+13$ & & $<2.6 \mathrm{E}-08$ & \\
\hline WD1040+492 & 230 & 30.3 & 3.7 & $2.4 \mathrm{E}+13$ & $2.9 \mathrm{E}+12$ & $2.6 \mathrm{E}-08$ & $3.2 \mathrm{E}-09$ \\
\hline WD1041+580 & 93 & 14.6 & & $<1.2 \mathrm{E}+13$ & & $<3.1 \mathrm{E}-08$ & \\
\hline WD1057+719 & 141 & 26.3 & 2.9 & $2.1 \mathrm{E}+13$ & $2.3 \mathrm{E}+12$ & $3.7 \mathrm{E}-08$ & $4.1 \mathrm{E}-09$ \\
\hline WD1109-225 & 82 & 13.8 & 4.0 & $1.1 \mathrm{E}+13$ & $3.2 \mathrm{E}+12$ & $3.4 \mathrm{E}-08$ & $9.7 \mathrm{E}-09$ \\
\hline WD1234+481 & 129 & 10.4 & 3.0 & $8.3 \mathrm{E}+12$ & $2.4 \mathrm{E}+12$ & $1.6 \mathrm{E}-08$ & $4.6 \mathrm{E}-09$ \\
\hline WD1254+223 & 67 & 15.1 & 5.0 & $1.2 \mathrm{E}+13$ & $4.0 \mathrm{E}+12$ & $4.5 \mathrm{E}-08$ & $1.5 \mathrm{E}-08$ \\
\hline WD1302+597 & 79 & 17.4 & & $<1.4 \mathrm{E}+13$ & & $<4.4 \mathrm{E}-08$ & \\
\hline WD1314+293 & 68 & 10.5 & 1.8 & $8.4 \mathrm{E}+12$ & $1.4 \mathrm{E}+12$ & $3.1 \mathrm{E}-08$ & $5.3 \mathrm{E}-09$ \\
\hline WD1337+701 & 104 & 113.4 & & $<9.1 \mathrm{E}+13$ & & $<2.2 \mathrm{E}-07$ & \\
\hline WD1440+753 & 98 & 11.9 & & $<9.5 \mathrm{E}+12$ & & $<2.4 \mathrm{E}-08$ & \\
\hline WD1528+487 & 140 & 23.8 & 4.1 & $1.9 \mathrm{E}+13$ & $3.3 \mathrm{E}+12$ & $3.4 \mathrm{E}-08$ & $5.8 \mathrm{E}-09$ \\
\hline WD1550+130 & 756 & 23.0 & & $<1.8 \mathrm{E}+13$ & & $<6.1 \mathrm{E}-09$ & \\
\hline WD1603+432 & 114 & 16.1 & & $<1.3 \mathrm{E}+13$ & & $<2.8 \mathrm{E}-08$ & \\
\hline WD1615-154 & 55 & 12.0 & & $<9.6 \mathrm{E}+12$ & & $<4.4 \mathrm{E}-08$ & \\
\hline WD1620+647 & 174 & 17.2 & & $<1.4 \mathrm{E}+13$ & & $<2.0 \mathrm{E}-08$ & \\
\hline WD1631+781 & 67 & 6.9 & 2.1 & $5.5 \mathrm{E}+12$ & $1.6 \mathrm{E}+12$ & $2.0 \mathrm{E}-08$ & $6.2 \mathrm{E}-09$ \\
\hline WD1635+529 & 123 & 16.3 & & $<1.3 \mathrm{E}+13$ & & $<2.7 \mathrm{E}-08$ & \\
\hline WD1636+351 & 109 & 15.1 & & $<1.2 \mathrm{E}+13$ & & $<2.8 \mathrm{E}-08$ & \\
\hline WD1648+407 & 200 & 21.9 & 9.3 & $1.7 \mathrm{E}+13$ & $7.4 \mathrm{E}+12$ & $2.2 \mathrm{E}-08$ & $9.3 \mathrm{E}-09$ \\
\hline WD1725+586 & 123 & 53.1 & 5.2 & $4.2 \mathrm{E}+13$ & $4.1 \mathrm{E}+12$ & $8.6 \mathrm{E}-08$ & $8.4 \mathrm{E}-09$ \\
\hline WD1800+685 & 159 & 11.7 & 2.1 & $9.4 \mathrm{E}+12$ & $1.7 \mathrm{E}+12$ & $1.5 \mathrm{E}-08$ & $2.7 \mathrm{E}-09$ \\
\hline WD1819+580 & 103 & 13.7 & & $<1.1 \mathrm{E}+13$ & & $<2.7 \mathrm{E}-08$ & \\
\hline WD1844-223 & 62 & 7.4 & & $<5.9 \mathrm{E}+12$ & & $<2.4 \mathrm{E}-08$ & \\
\hline WD1845+683 & 125 & 32.2 & & $<2.6 \mathrm{E}+13$ & & $<5.2 \mathrm{E}-08$ & \\
\hline WD1917+509 & 105 & 12.5 & & $<9.9 \mathrm{E}+12$ & & $<2.4 \mathrm{E}-08$ & \\
\hline WD1921-566 & 110 & 22.2 & & $<1.8 \mathrm{E}+13$ & & $<4.0 \mathrm{E}-08$ & \\
\hline WD1950-432 & 140 & 17.5 & 5.0 & $1.4 \mathrm{E}+13$ & $3.9 \mathrm{E}+12$ & $2.5 \mathrm{E}-08$ & 7.1E-09 \\
\hline WD2004-605 & 58 & 9.4 & 2.8 & $7.5 \mathrm{E}+12$ & $2.2 \mathrm{E}+12$ & $3.3 \mathrm{E}-08$ & $9.6 \mathrm{E}-09$ \\
\hline WD2014-575 & 51 & 29.0 & & $<2.3 \mathrm{E}+13$ & & $<1.1 \mathrm{E}-07$ & \\
\hline WD2020-425 & 52 & 42.0 & & $<3.4 \mathrm{E}+13$ & & $<1.6 \mathrm{E}-07$ & \\
\hline WD2116+736 & 177 & 9.9 & 2.6 & $7.9 \mathrm{E}+12$ & $2.1 \mathrm{E}+12$ & $1.1 \mathrm{E}-08$ & $2.9 \mathrm{E}-09$ \\
\hline WD2124+191 & 46 & 44.7 & & $<3.6 \mathrm{E}+13$ & & $<1.9 \mathrm{E}-07$ & \\
\hline WD2124-224 & 224 & 20.1 & 4.7 & $1.6 \mathrm{E}+13$ & $3.7 \mathrm{E}+12$ & $1.8 \mathrm{E}-08$ & $4.2 \mathrm{E}-09$ \\
\hline WD2152-548 & 129 & 90.0 & 6.6 & $7.2 \mathrm{E}+13$ & $5.2 \mathrm{E}+12$ & $1.4 \mathrm{E}-07$ & $1.0 \mathrm{E}-08$ \\
\hline WD2257-073 & 89 & 19.1 & 6.2 & $1.5 \mathrm{E}+13$ & $4.9 \mathrm{E}+12$ & $4.3 \mathrm{E}-08$ & $1.4 \mathrm{E}-08$ \\
\hline WD2309+105 & 79 & 5.1 & & $<4.1 \mathrm{E}+12$ & & $<1.3 \mathrm{E}-08$ & \\
\hline WD2321-549 & 192 & 22.3 & 6.3 & $\sim 1.8 \mathrm{E}+13$ & & $\sim 2.3 \mathrm{E}-08$ & \\
\hline WD2331-475 & 82 & 6.6 & 2.4 & $\sim 5.3 \mathrm{E}+12$ & & $\sim 1.6 \mathrm{E}-08$ & \\
\hline WD2350-706 & 92 & 22.6 & 3.6 & $\sim 1.8 \mathrm{E}+13$ & & $\sim 4.9 \mathrm{E}-08$ & \\
\hline
\end{tabular}

Notes. We also give the estimated distance to each star from which the volume densities are calculated. Included in the sample are the upper limits $(3 \sigma)$ on column density for those white dwarfs where interstellar material is not detected but where the spectra are of sufficient signal-to-noise to obtain meaningful limits on the amount of $\mathrm{O}$ vi present. Blank spaces in the error columns denote that a density value is an upper limit. Symbol " " indicates that there is significant systematic uncertainty in this measurement due to the blending of interstellar and photospheric components. 


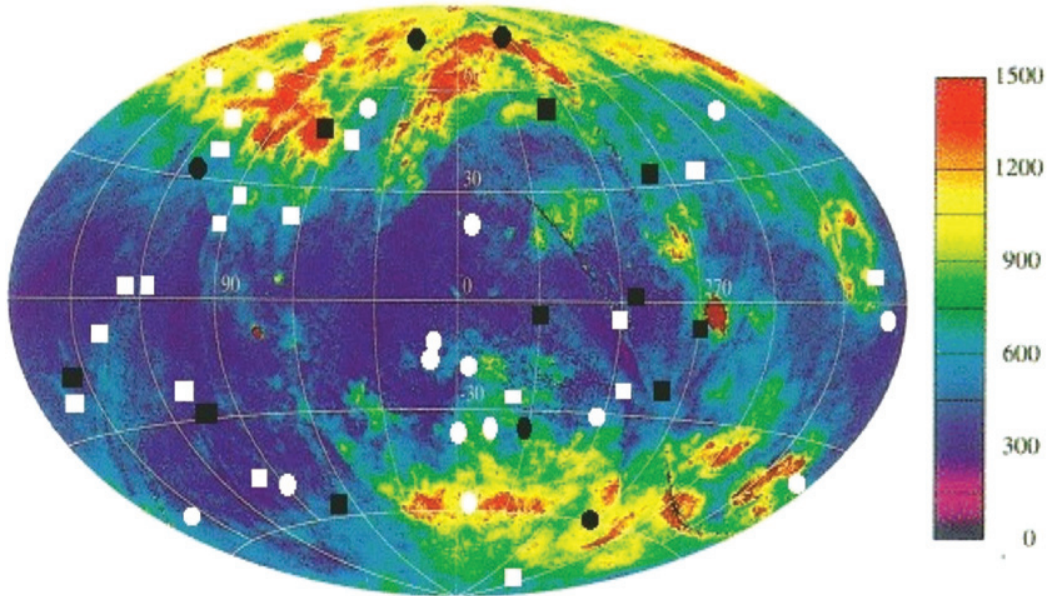

Figure 7. Location of detections and non-detections of O VI absorption toward the hot white dwarfs in this study, seven B-stars from Welsh \& Lallement (2008) and eight B-stars from Bowen et al. (2008) superimposed on a map of the spatial distribution of the SXRB emission intensity (Snowden et al. 1997). Symbols are filled black circles, $\mathrm{O}$ vi detections below $80 \mathrm{pc}$; filled white circles, non-detections below $80 \mathrm{pc}$; filled black squares, detections between 80 and $120 \mathrm{pc}$; filled white squares, non-detections between 80 and $120 \mathrm{pc}$. The color bar to the right of the map is the key to the intensity of the SXRB in units of $10^{-6} \mathrm{counts} \mathrm{s}^{-1}$ arcmin ${ }^{-2}$.

(A color version of this figure is available in the online journal.)

Table 6

Key for Figure 8

\begin{tabular}{|c|c|c|c|c|c|c|c|c|}
\hline WD & Star & $v_{\text {is }}$ inspection & Distance & $l$ & $b$ & $N_{\mathrm{O} \text { vI }}$ & $\log \left(N_{\mathrm{O}}{ }_{\mathrm{VI}}\right)$ & Map Index \\
\hline WD1844-223 & REJ, EUVEJ & ND & 62.00 & 12.5022 & -9.2474 & $<5.9 \mathrm{e} 12$ & $<12.77$ & 1 \\
\hline WD2124-224 & REJ, EUVEJ & I & 224.00 & 27.3595 & -43.7596 & $1.6 \mathrm{e} 13$ & 13.20 & 2 \\
\hline WD1314+293 & HZ43 & IR & 68.00 & 54.1058 & 84.1621 & $8.4 \mathrm{e} 12$ & 12.92 & 3 \\
\hline WD1648+407 & REJ, EUVEJ & I & 200.00 & 64.6411 & 39.6014 & $1.7 \mathrm{e} 13$ & 13.23 & 4 \\
\hline WD2257-073 & HD217411 & IR & 88.50 & 65.1744 & -56.9335 & $1.5 \mathrm{e} 13$ & 13.18 & 5 \\
\hline WD1528+487 & REJ, EUVEJ & I & 140.00 & 78.8701 & 52.7205 & $1.9 \mathrm{e} 13$ & 13.28 & 6 \\
\hline WD1725+586 & LB335 & IR & 123.20 & 87.1717 & 33.8299 & $4.2 \mathrm{e} 13$ & 13.62 & 7 \\
\hline WD2309+105 & GD246 & ND & 79.00 & 87.2625 & -45.1174 & $<4.1 \mathrm{e} 12$ & $<12.61$ & 8 \\
\hline WD1800+685 & KUV & I & 159.00 & 98.73 & 29.7751 & $9.4 \mathrm{e} 12$ & 12.97 & 9 \\
\hline WD2116+736 & KUV & I & 177.00 & 109.3900 & 16.9240 & $7.9 \mathrm{e} 12$ & 12.90 & 10 \\
\hline WD1631+781 & REJ, EUVEJ & I & 67.00 & 111.2953 & 33.5782 & $5.5 \mathrm{e} 12$ & 12.74 & 11 \\
\hline WD0004+330 & GD2 & I & 98.80 & 112.4803 & -28.6882 & $4.9 \mathrm{e} 12$ & 12.69 & 12 \\
\hline WD1234+481 & PG & I & 129.00 & 129.8100 & 69.0086 & $8.3 \mathrm{e} 12$ & 12.92 & 13 \\
\hline WD1057+719 & PG & IR & 141.00 & 134.4790 & 42.9215 & $2.1 \mathrm{e} 13$ & 13.32 & 14 \\
\hline WD1040+492 & REJ, EUVEJ & IR & 230.00 & 162.6693 & 57.0087 & $2.4 \mathrm{e} 13$ & 13.38 & 15 \\
\hline WD0937+505 & PG & I & 218.00 & 166.9021 & 47.1203 & $3.2 \mathrm{e} 13$ & 13.51 & 16 \\
\hline WD0235-125 & PHL1400 & ND & 86.20 & 187.3990 & -61.1220 & $<1.6 \mathrm{e} 13$ & $<13.20$ & 17 \\
\hline WD0346-011 & GD50 & ND & 29.00 & 188.9534 & -40.0974 & $<4.5 \mathrm{e} 12$ & $<12.65$ & 18 \\
\hline WD0549+158 & GD71 & ND & 49.00 & 192.0286 & -5.3382 & $<3.2 \mathrm{e} 12$ & $<12.51$ & 19 \\
\hline WD0455-282 & REJ, EUVEJ & $\mathrm{IB}+\mathrm{P}$ & 102.00 & 229.2948 & -36.1650 & $9.0 \mathrm{e} 12$ & 12.95 & 20 \\
\hline WD0229-481 & REJ, EUVEJ & ND & 239.60 & 266.6179 & -61.5914 & $<9.6 \mathrm{e} 12$ & $<12.98$ & 21 \\
\hline WD0320-539 & LB1663 & ND & 124.10 & 267.3001 & -51.6355 & $<1.3 \mathrm{e} 13$ & $<13.11$ & 22 \\
\hline WD0830-535 & REJ, EUVEJ & I & 82.00 & 270.1121 & -8.2658 & $2.5 \mathrm{e} 13$ & 13.40 & 23 \\
\hline WD1109-225 & HD97277 & I & 81.56 & 274.7780 & 34.5360 & $1.1 \mathrm{e} 13$ & 13.04 & 24 \\
\hline WD0715-704 & REJ, EUVEJ & I & 94.00 & 281.4000 & -23.5000 & $9.2 \mathrm{e} 12$ & 12.96 & 25 \\
\hline WD0325-857 & REJ, EUVEJ & ND & 35.00 & 299.8575 & -30.6815 & $<4.2 \mathrm{e} 12$ & $<12.62$ & 26 \\
\hline WD0027-636 & REJ, EUVEJ & $\mathrm{I}+\mathrm{P}$ & 236.00 & 306.9798 & -53.5497 & $\sim 1.1 \mathrm{e} 13$ & $\sim 13.04$ & 27 \\
\hline WD2350-706 & HD223816 & $\mathrm{I}+\mathrm{P}$ & 92.00 & 309.9147 & -45.9375 & $\sim 1.8 \mathrm{e} 13$ & $\sim 13.26$ & 28 \\
\hline WD1254+223 & GD153 & IR & 67.00 & 317.2548 & 84.7465 & $1.2 \mathrm{e} 13$ & 13.08 & 29 \\
\hline WD2321-549 & REJ, EUVEJ & $\mathrm{I}+\mathrm{P}$ & 192.00 & 326.9080 & -58.2104 & $\sim 1.8 \mathrm{e} 13$ & $\sim 13.26$ & 30 \\
\hline WD2331-475 & REJ, EUVEJ & $\mathrm{I}+\mathrm{P}$ & 82.00 & 334.8358 & -64.8080 & $\sim 5.3 \mathrm{e} 12$ & $\sim 12.72$ & 31 \\
\hline WD2004-605 & REJ, EUVEJ & I & 58.00 & 336.5813 & -32.8586 & $7.5 \mathrm{e} 12$ & 12.88 & 32 \\
\hline WD2014-575 & LS210-114 & ND & 51.00 & 340.2003 & -34.2481 & $<2.3 \mathrm{e} 13$ & $<13.36$ & 33 \\
\hline WD1950-432 & HS & I & 140.00 & 356.5050 & -29.0920 & $1.4 \mathrm{e} 13$ & 13.15 & 34 \\
\hline WD2020-425 & REJ, EUVEJ & ND & 52.20 & 358.3597 & -34.4540 & $<3.4 \mathrm{e} 13$ & $<13.53$ & 35 \\
\hline WD1615-154 & G153-41 & ND & 55.00 & 358.7916 & 24.1800 & $<9.6 \mathrm{e} 12$ & $<12.98$ & 36 \\
\hline
\end{tabular}

Note. Symbol " " indicates that there is significant systematic uncertainty in this measurement due to the blending of interstellar and photospheric components.

The origin of this infalling gas was thought to be part of the Intermediate Velocity Arch feature that resides in the overlying galactic halo. However as stated above, the recent work of
Wolleben (2007) argues in favor of an interaction between gas ejected from the adjacent interstellar cavity of Loop I and the Local Cavity. 

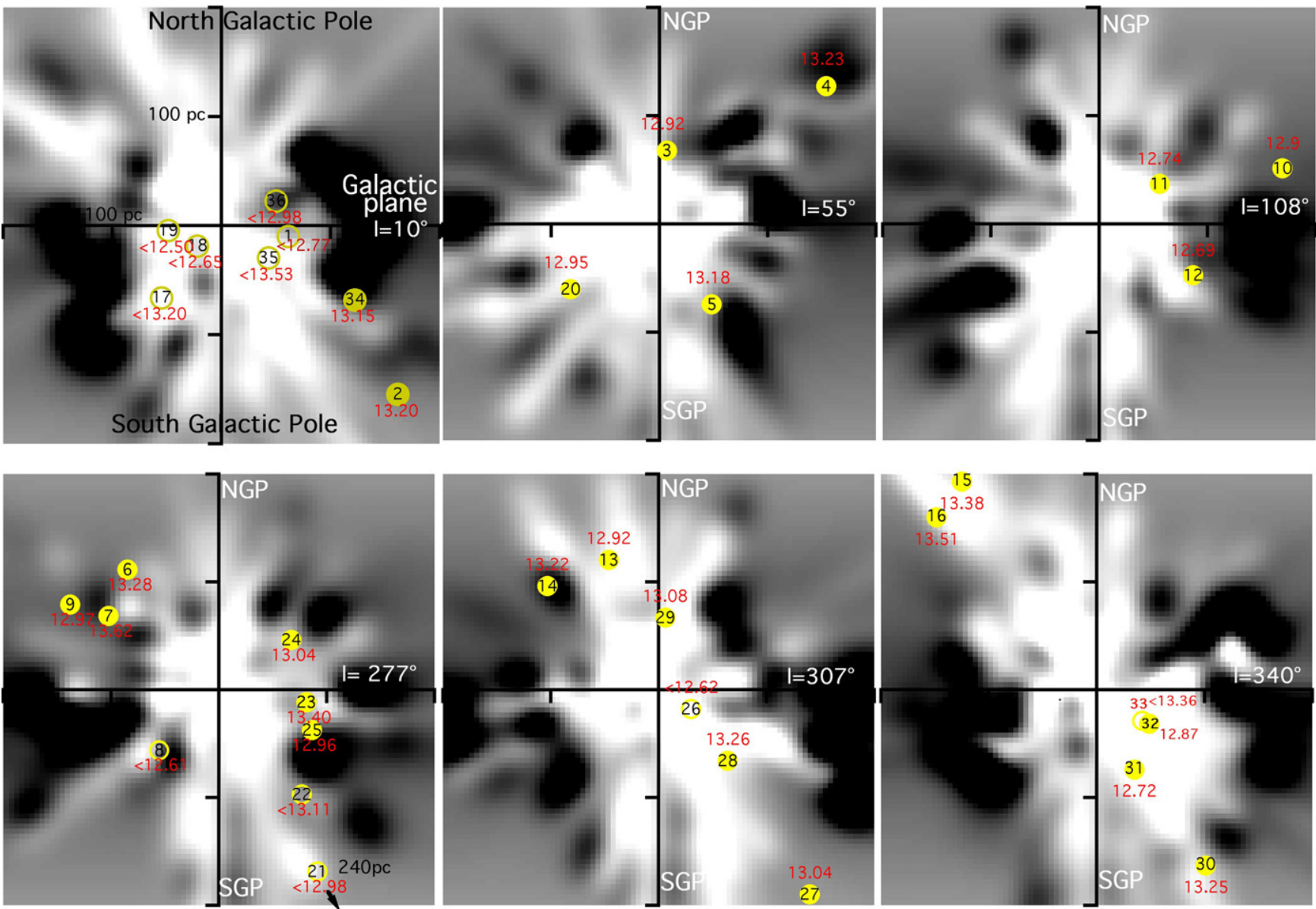

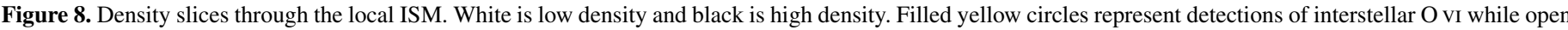

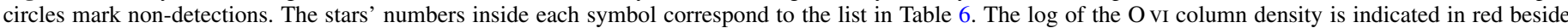

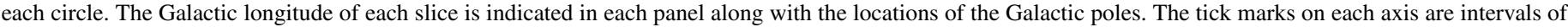
$100 \mathrm{pc}$ distance from the Sun. We note that star 21 in the $l=277^{\circ}$ slice actually lies off the map in the direction of the arrow at a distance of $240 \mathrm{pc}$.

(A color version of this figure is available in the online journal.)

\section{CONCLUSIONS}

We have carried out a comprehensive survey of O vi absorption in the local ISM using the large sample of white dwarf spectra available in the FUSE data archive. Since the FUSE mission has now ended and in the absence of any plans for a future space facility covering the same wavelength range, this work will provide the most complete analysis for the foreseeable future. In total, this work covers 80 lines of sight, with a sensitivity varying according to the brightness of the background white dwarf and the exposure time allocated. Hence, we have expanded by factors of 3.6 and 1.7, respectively, the number of sight lines included in the earlier work of Oegerle et al. (2005) and Savage \& Lehner (2006).

We have detected interstellar O VI along 26 lines of sight in this sample. In most of these, the O vi velocity is close to that of the neutral ISM, but one star has $\mathrm{O}$ vi blueshifted relative to the ISM while a few others have redshifted components. There are very few detections of $\mathrm{O}$ VI within the inner rarefied regions of the LB, with the nearest detection of interstellar $\mathrm{O}$ vi being toward WD2004-605 at a distance of $58 \mathrm{pc}$. The derived upper limits to detections within the bubble provide tight constraints on the amount of hot gas that might be present.

In support of previous observations, we find that the spatial distribution of $\mathrm{O}$ VI is best described as being "patchy" within 200 pc. Although O VI has been detected along sight lines at high galactic latitudes and toward regions of enhanced SXRB flux, there are many more cases where this is not the case. Therefore, we find no strong evidence to support a spatial correlation between $\mathrm{O}$ VI and SXRB emission, thus refuting the initial findings of Welsh \& Lallement (2008). However, we find that the majority of white dwarfs yielding interstellar detections of $\mathrm{O}$ VI are mostly located close to or beyond the neutral boundary to the Local Cavity in regions of higher gas density. Hence, any $T \sim 300,000 \mathrm{~K}$ gas responsible for the $\mathrm{O}$ VI absorption may reside at the interface between the cavity and surrounding medium or in that medium itself.

This work is based on data from the NASA-CNES-CSA FUSE mission, operated by Johns Hopkins University. The data were obtained from the MAST archive operated by the Space Telescope Science Institute. M.A.B. and D.D.B. acknowledge the support of the Science and Technology Facilities Council (UK).

\section{APPENDIX}

\section{COMMENTS ON INDIVIDUAL STARS AND LINES OF SIGHT}

In this section, we provide individual comments about some of the stars and the interstellar absorption observed. Measurements associated with these objects are summarized in Tables 3-5. 
WD0027-636: strong Si IV and P v. The O vi feature is flatbottomed in this object and spans both photospheric and interstellar velocities. If the feature is purely stellar, it is asymmetric compared to the stellar velocity. Therefore, this appears to have blended, but unresolved, interstellar and photospheric components.

WD0050-332: although this is a relatively cool DA at $34,684 \mathrm{~K}$, photospheric material has been detected in the STIS spectrum (Barstow et al. 2003b; Bannister et al. 2003) and P V is present in the FUSE spectrum. The $\mathrm{O}$ VI is clearly aligned with the stellar velocity.

WD0131-164: detections of photospheric Si IV and Pv (see Figure 1). The $\mathrm{O}$ vI velocity is aligned with these velocities.

WD0026-615: detections of photospheric Si IV and P IV. The $\mathrm{O}$ VI velocity is aligned with these velocities.

WD0232+035: Feige 24 is a well-studied hot DA+dM binary system known to contain photospheric heavy elements, also detected in the FUSE spectrum (e.g., Si IV and P v). The photospheric velocity varies with phase about the $\sim 4.25 \mathrm{~d}$ period. In this observation, the photospheric and interstellar components are not resolved, so the $\mathrm{O}$ VI is assumed to be photospheric.

WD0252-055: detection of photospheric Si IV and P IV. ISM velocity aligned with stellar velocity. Therefore, $\mathrm{O}$ vI is assumed to be a stellar component.

WD0353+284: O VI component is at a velocity redshifted when compared to the interstellar velocity.

WD0455-282: a complex line of sight with two interstellar velocity components (see Table 3 ). There are both photospheric and interstellar O VI components, with the latter spanning the two interstellar lines.

WD0512+326: detection of photospheric Si IV. The O VI velocity is aligned with these velocities.

WD0603-483: there are two interstellar velocity components as noted in Table 3, clearly detected in C II, but also weakly seen in $\mathrm{O}$ I.

WD0621-376: strong detections of photospheric material in both IUE (e.g., Holberg et al. 1998) and FUSE. The O vI is aligned with the photospheric velocity although the photospheric and interstellar components are not clearly resolved.

WD0715-704: the O VI velocity is aligned with the interstellar velocity. No photospheric material is detected in this star.

WD0802+413: two interstellar components are detected in this star, as detailed in Table 3, but the weaker (at $\sim 70 \mathrm{~km} \mathrm{~s}^{-1}$ ) is seen only in C II.

WD1021+266: two interstellar components are detected in this star, as detailed in Table 3, but the weaker (at $\sim 50 \mathrm{~km} \mathrm{~s}^{-1}$ ) is seen only in C II. Photospheric C III, Si IV, and S IV are detected at $13.8 \mathrm{~km} \mathrm{~s}^{-1}$. The $\mathrm{O}$ vi velocity is aligned with this velocity.

WD1029+537: photospheric C III, Si IV, S VI, and PV are detected at $12.0 \mathrm{~km} \mathrm{~s}^{-1}$. The $\mathrm{O}$ vI velocity is aligned with this velocity.

WD1040+492: multiple interstellar components detected in both $\mathrm{O}$ I and $\mathrm{C}$ II. The components are resolved in $\mathrm{O}$ I but blended and saturated in $\mathrm{C}$ II.

WD1057+719: the OI is contaminated by a geocoronal emission line blueward of the absorption core. The $\mathrm{O}$ VI velocity is redward of the ISM value.

WD1254+223: the O VI absorption is board overlapping with the interstellar velocity but redward of a centroid of $17.4 \mathrm{~km} \mathrm{~s}^{-1}$.

WD1302+597: the O I interstellar line is completely filled in by geocoronal emission, but $\mathrm{C}$ II is detected.

WD1314+293: the O VI absorption is board overlapping with the interstellar velocity but redward of a centroid of $19.8 \mathrm{~km} \mathrm{~s}^{-1}$.
This star and WD1254+223 both lie near the north Galactic pole.

WD1550+130: two ISM components are present. The weaker of the two at $43.8 \mathrm{~km} \mathrm{~s}^{-1}$ is only detected in the $\mathrm{C}_{\text {II. }}$.

WD1611-084: photospheric material has been detected in the HST/GHRS spectrum of this star (e.g., Barstow et al. 2003). The photospheric and ISM velocities are not well separated. Therefore, the $\mathrm{O} v \mathrm{vI}$ is taken to be photospheric. There are two interstellar $\mathrm{C}_{\text {II }}$ components present.

WD1620+64: the C II interstellar line has a strongly asymmetric profile and extended red wing, indicating that more than one component may be present. The $\mathrm{O}$ I line is filled in by geocoronal emission.

WD1636+351: there are two interstellar components at -31.1 and $24.9 \mathrm{~km} \mathrm{~s}^{-1}$. The weaker redward component is not detected in $\mathrm{O}$.

WD1725+586: there are at least two strong, saturated components detected in $\mathrm{C}$ II, which are barely resolved. No O I is detected as the feature is filled in by strong geocoronal emission. The detected O VI component coincides with the redward ISM line and there may be a weaker $\mathrm{O} v \mathrm{VI}$ line aligned with the blueward ISM component. However, this falls below our detection threshold. The $1037 \AA \mathrm{O}$ vI line is also detected in this star with a measured column density of $7.3 \times 10^{-13} \mathrm{~cm}^{-2}$, almost twice that of the $1031 \AA$ line. This suggests that the 1031 line may be saturated. Since the velocities of the two lines are in very close agreement, this could be a rare example of unresolved interstellar $\mathrm{O}$ VI tracing a temperature far out of equilibrium.

WD1845+683: both $\mathrm{C}_{\mathrm{II}}$ and $\mathrm{O}$ I are detected but the $\mathrm{O}_{\mathrm{I}}$ is contaminated by a geocoronal line.

WD1942+499: Si IV and P V are detected in this star and the $\mathrm{O}$ VI detected is clearly aligned with this velocity component rather than the $\mathrm{C}$ II and $\mathrm{O}$.

WD1950-432: while photospheric material (P IV, Si IV, S IV) is identified in this star, the O VI velocity is well aligned with the interstellar velocity.

WD2000-561: stellar Si IV and PIV are detected. The measured $O$ vi velocity lies between the interstellar and photospheric velocities. Therefore, the $\mathrm{O}$ vi could be photospheric. The interstellar $\mathrm{O}_{\mathrm{I}}$ is contaminated by a geocoronal contribution.

WD2011+398: stellar S IV and PIV are detected. The measured $O$ vi velocity aligns with the photospheric value.

WD2146-433: stellar S IV and PIV are detected. The measured $\mathrm{O}$ vi velocity aligns with the photospheric value.

WD2321-549, WD2331-475, WD2350-706: the O VI absorption feature is very broad in all three of these stars, spans both interstellar and photospheric velocities, and appears to be a multiple, although not resolved component.

\section{REFERENCES}

Bannister, N. P., Barstow, M. A., Holberg, J. B., \& Bruhweiler, F. C. 2003, MNRAS, 341, 477

Barstow, M. A., Bond, H. E., Burleigh, M. R., \& Holberg, J. B. 2001, MNRAS, 322, 891

Barstow, M. A., Bond, H. E., Holberg, J. B., Burleigh, M. R., Hubeny, I., \& Koester, D. 2005, MNRAS, 362, 1134

Barstow, M. A., Dobbie, P. D., Forbes, A. E., \& Boyce, D. D. 2007, in ASP Conf. Ser. 372, 15th European Workshop on White Dwarfs, ed. R. Napiwotzki \& M. R. Burleigh (San Francisco, CA: ASP), 243

Barstow, M. A., Good, S. A., Burleigh, M. R., Hubeny, I., Holberg, J. B., \& Levan, A. 2003a, MNRAS, 344, 562

Barstow, M. A., Good, S. A., Holberg, J. B., Hubeny, I., Bannister, N. P., Bruhweiler, F. C., Burleigh, M. R., \& Napiwotzki, R. 2003b, MNRAS, 341, 870 
Barstow, M. A., Jordan, S., O’Donoghue, D., Burleigh, M. R., Napiwotzki, R., \& Harrop-Allin, M. K. 1995, MNRAS, 277, 971

Barstow, M. A., et al. 1994, MNRAS, 271, 175

Bowen, D., et al. 2008, ApJS, 176, 59

Bowyer, C. S., Field, G. B., \& Mack, J. F. 1968, Nature, 217, 32

Boyce, D. D. 2009, PhD thesis, Univ. Leicester

Burleigh, M. R., Barstow, M. A., \& Fleming, T. A. 1997, MNRAS, 287, 381

Christian, D. J., Vennes, S., Thorstensen, J. R., \& Mathioudakis, M. 1996, AJ, 112,258

Cox, D. P. 1998, in IAU Colloq. 166, The Local Bubble and Beyond, ed. D. Breitschwerdt, M. J. Freyberg, \& J. Trümper (Lecture Notes in Physics, Vol. 506; Berlin: Springer), 121

Cox, D. P., \& Helenius, L. 2003, ApJ, 583, 205

Cravens, T. 1997, Geophys. Res. Lett., 24, 105

Cravens, T. 2000, ApJ, 532, L153

Crawford, I., et al. 2002, MNRAS, 337, 720

Dixon, W. V., Sankrit, R., \& Otte, B. 2006, ApJ, 647, 328

Dixon, W. V., et al. 2007, PASP, 119, 527

Dobbie, P. D., Barstow, M. A., Hubeny, I., Holberg, J. B., Burleigh, M. R., \& Forbes, A. E. 2005, MNRAS, 363, 763

Dupuis, J., Chayer, P., Vennes, S., Kruk, J. W., Torres, L. M., \& Sanchez, P. J. 2005, in ASP Conf. Ser. 334, 14th European Workshop on White Dwarfs, ed. D. Koester \& S. Moehler (San Francisco, CA: ASP), 191

Egger, R. 1998, in IAU Colloq. 166, The Local Bubble and Beyond, ed. D. Breitschwerdt, M. J. Freyberg, \& J. Trümper (Lecture Notes in Physics, Vol. 506; Berlin: Springer), 287

ESA 1997, The Hipparcos and Tycho Catalogues (ESA SP-1200; Noordwijk: ESA)

Finley, D. S., Koester, D., \& Basri, G. 1997, ApJ, 488, 375

Freyberg, M. J. 1994, PhD thesis, Technishe Univ., München

Heiles, C. 1998, ApJ, 498, 689

Holberg, J. B., Barstow, M. A., Bruhweiler, F. C., \& Hubeny, I. 2000, BAAS, 197,8304

Holberg, J. B., Barstow, M. A., Bruhweiler, F. C., Hubeny, I., \& Green, E. M. 1999, ApJ, 517, 870

Holberg, J. B., Barstow, M. A., Bruhweiler, F. C., \& Sion, E. M. 1995, ApJ, 453,313

Holberg, J. B., Barstow, M. A., Bruhweiler, F. C., \& Sion, E. M. 1997, ApJ, 453,313

Holberg, J. B., Barstow, M. A., Lanz, T., \& Hubeny, I. 1997, ApJ, 484, 871

Holberg, J. B., Barstow, M. A., \& Sion, E. M. 1998, ApJS, 119, 207

Holberg, J. B., Bergeron, P., \& Gianninas, A. 2008, AJ, 135, 1239

Hurwitz, M., Sasseen, T., \& Sirk, M. 2005, ApJ, 623, 911

Indebetouw, R., \& Shull, J. M. 2004, ApJ, 605, 205

Jenkins, E. B. 1978, ApJ, 219, 845

Jenkins, E. B., \& Meloy, D. A. 1974, ApJ, 193, 121

Koutroumpa, D., Lallement, R., Raymond, J. C., \& Kharchenko, V. 2009, ApJ, 696, 1517
Koutroumpa, D., et al. 2008, Space Sci. Rev., 143, 217

Kuntz, K., \& Snowden, S. 2000, ApJ, 543, 195

Kurucz, R, \& Bell, B 1995, Kuruzc CD-ROM no. 23 (Cambridge, MA: Smithsonian Astrophysical Observatory)

Lallement, R. 2004, A\&A, 418, 143

Lallement, R., Welsh, B. Y., Vergely, J. L., Crifo, F., \& Sfeir, D. 2003, A\&A, 411,447

Landsman, W., Simon, T. \& Bergeron, P. 1993, PASP, 105, 841

Lisse, C. M., et al. 1996, Science, 274, 205

Marsh, M. C., et al. 1997, MNRAS, 286, 369

Mathewson, D., \& Ford, V. 1970, MNRAS, 74, 139

McCammon, D., Burrows, D. N., Sanders, W. T., \& Kraushaar, W. L. 1983, ApJ, 269, 107

McCook, G. P., \& Sion, E. M. 1999, ApJS, 121, 1

Moos, H. W., et al. 2000, ApJ, 538, L1

Morton, D. C. 2003, ApJS, 149, 205

Oegerle, W. R., Jenkins, E. B., Shelton, R. L., Bowen, D. B., \& Chayer, P. 2005, ApJ, 622, 377

Redfield, S., \& Linsky, J. 2008, ApJ, 673, 283

Rogerson, J. B., York, D. G., Drake, J. F., Jenkins, E. B., Morton, D. C., \& Sptizer, L. 1973, ApJ, 181, 110

Sahnow, D. S., et al. 2000, ApJ, 538, L7

Sahu, M. S., et al. 1999, ApJ, 523, L156

Savage, B. D., \& Lehner, N. 2006, ApJS, 162, 134

Sfeir, D., Lallement, R., Crifo, F., \& Welsh, B. 1999, A\&A, 346, 785

Shelton, R. L. 1998, ApJ, 504, 785

Shelton, R. L. 1999, ApJ, 521, 217

Snowden, S., Egger, R., Finkbeiner, D., Freyberg, M., \& Plucinsky, P. 1998, ApJ, 493, 715

Snowden, S., Freyberg, M., Kuntz, K., \& Sanders, W. 2000, ApJS, 128, 171

Snowden, S., et al. 1995, ApJ, 454, 643

Snowden, S., et al. 1997, ApJ, 485, 125

Vallerga, J. 1998, ApJ, 497, 921

Vallerga, J., \& Slavin, J. 1998, in IAU Colloq. 166, The Local Bubble and Beyond, ed. D. Breitschwerdt, M. J. Freyberg, \& J. Trümper (Lecture Notes in Physics, Vol. 506; Berlin: Springer), 79

Vennes, S. 1999, ApJ, 525, 995

Vennes, S., Christian, D. J., \& Thorstensen, J. R. 1998, ApJ, 502, 763

Vennes, S., Thejll, P. A., Genova-Galvan, R., \& Dupuis, J. 1997, ApJ, 480, 714

Welsh, B. Y., \& Lallement, R. 2008, A\&A, 490, 707

Welsh, B. Y., Lallement, R., Vergely, J.-L., \& Raimond, S. 2010, A\&A, 510, A54

Welsh, B. Y., Sallmen, S., \& Lallement, R. 2004, A\&A, 414, 261

Welsh, B. Y., Sfeir, D., Sirk, M., \& Lallement, R. 1999, A\&A, 352, 308

Welsh, B. Y., \& Shelton, R. 2009, ApSS, 323, 1

West, R. G., Sims, M. R., \& Willingale, R. 1994, Planet. Space Sci., 42, 71

Wolleben, M. 2007, ApJ, 664, 349

York, D.G. 1974, ApJ, 193, 127 